Bull. Soc. math. France

131 (4), 2003, p. 553-585

\title{
CONDUCTEUR, DESCENTE ET PINCEMENT
}

\author{
PAR DANIEL FERRAND
}

\begin{abstract}
RÉSUMÉ. - Une somme amalgamée de schémas est décrite localement par un produit fibré d'anneaux. Ce texte donne un résultat global d'existence (§ 5.4) de schémas définis comme certaines sommes amalgamées et un procédé algébrique $(\S 2.2)$ pour décrire les modules sur produits fibrés d'anneaux correspondants.

Abstract (Conductor, Descent and Pinching). - This paper investigates some fiber products of rings, and dually some pushouts of schemes. The algebraic side is centered on the use of the conductor to solve some descent problems, problems which can be better reached by comparing suitable categories of modules on the fiber product of rings, with the fiber product of the similar categories for each of the factors. The main algebraic result $(\S 2.2)$ asserts that these categories are very close to be equivalent, and that they are indeed equivalent as long as one restricts to flat modules. The geometric side is concerned with the existence of schemes defined by pinching: starting with a scheme $X^{\prime}$, a closed subscheme $Y^{\prime}$ and a finite morphism $Y^{\prime} \rightarrow Y$, the "pinching construction" is intended to produce a scheme $X$ which is the pushout of $X^{\prime}$ along the morphism $Y^{\prime} \rightarrow Y$; such a scheme is proved to exist ( $\left.\$ 5.4\right)$ under the mild asumption that any finite set of points in $X^{\prime}$ (resp. in $Y$ ) is contained in an open affine subset of $X^{\prime}$ (resp. of $\left.Y\right)$.
\end{abstract}

\section{Table des matières}

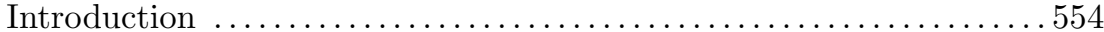

1. Produit fibré et somme amalgamée $\ldots \ldots \ldots \ldots \ldots \ldots \ldots \ldots \ldots \ldots 5$

2. Modules sur un produit fibré d'anneaux .................. 558

3. Traduction dans le langage de la théorie de la descente . . . . . . 563

Texte reçu le 16 décembre 2002, révisé le 17 mars 2003

Daniel Ferrand, Institut Mathématique de Rennes, Campus de Beaulieu, 35042 Rennes Cedex (France) • E-mail : ferrand@univ-rennes1.fr

Classification mathématique par sujets (2000). — 13Axx, 13Bxx, 14A15.

Mots clefs. - Produit fibré, somme amalgamée, descente finie, pincement. 


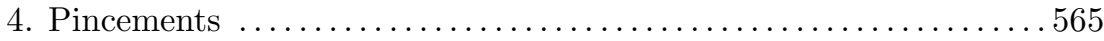

5. Existence de pincements; les cas utiles ..................567

6. Exemples de schémas propres et non projectifs ..............573

7. Existence de pincements; le cas général ....................5575

8. Applications aux monomorphismes .................... 579

Bibliographie .......................................584

\section{Introduction}

Ce travail(1) ${ }^{(1)}$ comporte un versant algébrique et un versant géométrique.

Il commence par l'étude des produits fibrés d'anneaux qui apparaissent lorsqu'un homomorphisme injectif $f: A \rightarrow A^{\prime}$ possède un conducteur non nul $I$ (c'est l'annulateur du $A$-module $A^{\prime} / A$ ). L'anneau $A$ est alors isomorphe au produit fibré $A^{\prime} \times_{A^{\prime} / I} A / I$, et il s'agit de relier certains énoncés portant sur $A$ à des énoncés analogues portant sur $A^{\prime}$ et sur $A / I$. La considération du conducteur est classiquement utilisée pour « descendre de $A^{\prime}$ à $A$ » une propriété d'un objet déjà défini sur $A$ (voir, parmi beaucoup d'autres, les démonstrations de [9], EGA $0_{\text {I }} 6.4 .9$, [9], EGA III 2.6.2 ou [8],...). Ici, on cherche plutôt à descendre l'objet lui-même, et non une de ses propriétés; le principal résultat (2.2) relie les modules sur le produit fibré $A$ et sur les facteurs $A^{\prime}$ et $A / I$; en particulier, on montre qu'une donnée de descente relative à $f$, sur un $A^{\prime}$-module $M^{\prime}$, est effective si elle le devient après réduction modulo le conducteur (3.1).

Restreint aux modules plats, cet énoncé peut être renforcé : la donnée d'un $A$-module plat $P$ est équivalente à la donnée d'un $A^{\prime}$-module plat $P^{\prime}$, d'un $A / I$ module plat $Q$ et d'un $A^{\prime} / I$-isomorphisme $A^{\prime} \otimes_{A} Q \stackrel{\sim}{\rightarrow} P^{\prime} / I P^{\prime}$. Ce résultat était déjà connu de Grothendieck lorsque le conducteur $I$ est nilpotent ( $c f$. [11] p. 45, lemme 11) et son analogue pour les modules projectifs de type fini est équivalent à un théorème de Milnor ( $c f .[3]$ p. 479 ou [12] p. 20).

Le point de vue géométrique conduit au problème du pincement : supposons toujours que $f$ soit injectif et posons $B=A / I$ et $B^{\prime}=A^{\prime} / I$, de sorte que $A$ s'identifie à l'anneau produit fibré $A^{\prime} \times_{B^{\prime}} B$. On montre (5.1) que le carré de morphismes de schémas

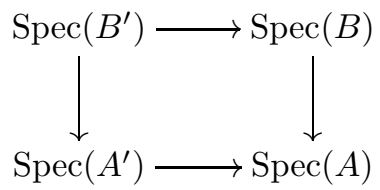

(1) Novembre 2002 : une première version de ce texte a été rédigée en mai et décembre 1970, et envoyée à ceux que je pensais intéressés; elle a fait partie d'une thèse soutenue en 1971 à Orsay, et n'a pas été autrement rendue publique. Pour la présente version, la plupart des démonstrations ont été profondément remaniées et quelques résultats ont été un peu complétés.

TOME $131-2003-\mathrm{N}^{\mathrm{O}} 4$ 
est cocartésien dans la catégorie des espaces annelés, autrement dit, que $\operatorname{Spec}(A)$ s'identifie à l'espace annelé somme amalgamée $\operatorname{Spec}\left(A^{\prime}\right) \sqcup_{\operatorname{Spec}\left(B^{\prime}\right)} \operatorname{Spec}(B)$.

Dans un langage plus géométrique - et imagé — on peut dire que $\operatorname{Spec}(A)$ est obtenu en «pinçant » $\operatorname{Spec}\left(A^{\prime}\right)$ le long du fermé $\operatorname{Spec}\left(B^{\prime}\right)$ par le morphisme $\operatorname{Spec}\left(B^{\prime}\right) \rightarrow \operatorname{Spec}(B)$.

Ces considérations gardent un sens pour des schémas qui ne sont plus affines mais c'est alors l'existence du schéma «pincé » qui pose un problème. D'ailleurs, on se heurte ici à un cas particulier de passage au quotient par une relation d'équivalence non plate et, comme on sait, dans la catégorie des schémas, l'existence de tels quotients est le plus souvent problématique.

Précisons ce qu'on entend par pincement : soient $Y^{\prime}$ un sous-schéma fermé d'un schéma $X^{\prime}$ et $g: Y^{\prime} \rightarrow Y$ un morphisme affine. Il existe toujours un espace annelé $X=X^{\prime} \sqcup_{Y^{\prime}} Y$ s'insérant dans un carré

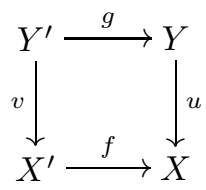

qui est cocartésien dans la catégorie des espaces annelés. On dit qu'on peut pincer $X^{\prime}$ le long de $Y^{\prime}$ par $g$ si $X$ est un schéma, si $f$ est affine et si u est une immersion fermée.

On donne en 5.4 et 7.1 des critères pour qu'il en soit ainsi; si on suppose de plus que $g$ est entier, la condition a une allure familière : pour tout $y \in Y, g^{-1}(y)$ doit être contenu dans un ouvert affine de $X^{\prime}$.

On retrouve, en particulier, la construction bien connue (cf. [19]) lorsque $X^{\prime}$ est une courbe projective, $Y^{\prime}$ un nombre fini de points de $X^{\prime}-$ ou, mieux, un diviseur - et $Y$ le corps de base.

Il faut mentionner que dans la catégorie des espaces algébriques (au sens de M. Artin), et lorsque $g$ est fini, l'existence de l'espace pincé ne requiert aucune hypothèse supplémentaire (voir [2] p. 120, th. 6.1).

\section{Produit fibré et somme amalgamée}

1.1. Un carré commutatif de flèches d'une catégorie $\mathcal{C}$

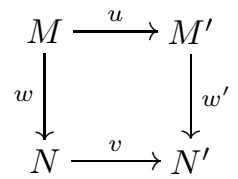

BULLETIN DE LA SOCIÉTÉ MATHÉMATIQUE DE FRANCE 
est dit cartésien si $u$ et $w$ font de $M$ un produit fibré de $N$ par $M^{\prime}$ au-dessus de $N^{\prime}$, c'est-à-dire si pour tout carré commutatif de $\mathcal{C}$ de la forme

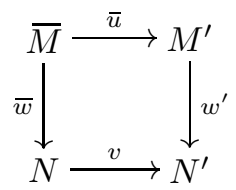

il existe une flèche et une seule $t: \bar{M} \rightarrow M$ telle que $\bar{u}=u t$ et $\bar{w}=w t$. On désignera alors souvent $M$ par le symbole $N \times_{N^{\prime}} M^{\prime}$.

On définit dualement la notion de carré cocartésien et de somme amalgamée; si le carré (1.1.1) est cocartésien, on désignera souvent $N^{\prime}$ par le symbole $N \sqcup_{M} M^{\prime}$.

Si $\mathcal{C}$ est la catégorie des ensembles et si (1.1.1) est cartésien, l'application $M \rightarrow N \times M^{\prime}$ déduite de $w$ et de $u$ induit une bijection de $M$ sur le sousensemble formé des couples $\left(y, x^{\prime}\right)$ tels que $v(y)=w^{\prime}\left(x^{\prime}\right)$.

Si $\mathcal{C}$ est la catégorie des modules sur un anneau fixé, ou la catégorie des anneaux, le carré ci-dessus est cartésien si et seulement si le carré d'ensembles sous-jacents l'est.

On déduit immédiatement du lemme du serpent le critère suivant :

Lemme 1.2. - Supposons que (1.1.1) désigne un carré commutatif dans la catégorie $\operatorname{Mod}(A)$ des modules sur un anneau commutatif $A$. Pour que ce carré soit cartésien dans $\operatorname{Mod}(A)$, il faut et il suffit que u induise un isomorphisme de $\operatorname{Ker}(w)$ sur $\operatorname{Ker}\left(w^{\prime}\right)$ et que $v$ induise une application injective de $\operatorname{Coker}(w)$ dans Coker $\left(w^{\prime}\right)$.

Lemme 1.3. - Considérons un carré cartésien

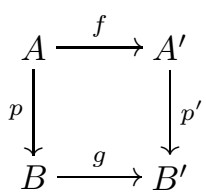

d'homomorphismes d'anneaux dans lequel $p^{\prime}$ est surjectif. Alors :

a) L'homomorphisme $p$ est surjectif.

b) En posant $I=\operatorname{Ker}(p), f$ induit une bijection de $I$ sur $f(I)=\operatorname{Ker}\left(p^{\prime}\right)$; en particulier, $f(I) A^{\prime}=f(I)$.

c) Les homomorphismes $g$ et $p^{\prime}$ permettent d'identifier $B^{\prime}$ et $B \otimes_{A} A^{\prime}$.

Inversement, soient $f: A \rightarrow A^{\prime}$ un homomorphisme d'anneaux et $I$ un idéal de $A$ tel que $f(I)$ soit un idéal de $A^{\prime}$ (c'est le cas si $I$ est le conducteur de $f$, 
c'est-à-dire l'idéal $\left.\operatorname{Ann}_{A}(\operatorname{Coker}(f))\right)$. Si $\operatorname{Ker}(f) \cap I=0$, alors le carré

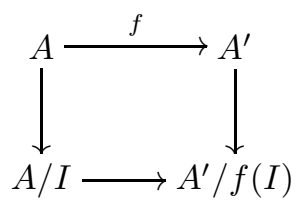

est cartésien.

1.3.1. Un cas particulier de ce diagramme a été popularisé sous le nom anglais de «D+M construction» : cela désigne la somme d'un idéal maximal $M$ d'un anneau (le plus souvent intègre) $T$ et d'un sous-anneau $D \subset T$ tel que $D \cap M=0$; or, le carré

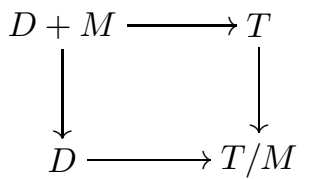

est alors cartésien.

1.3.2. Un autre cas particulier est connu sous le nom de «théorème chinois »; il s'énonce ainsi : si $I$ et $J$ sont des idéaux d'un anneau (commutatif) $R$, le carré suivant d'homomorphismes surjectifs est cartésien :

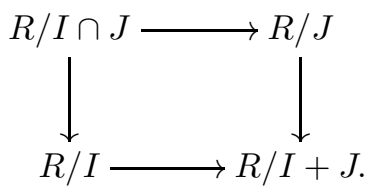

Lemme 1.4. - Soient $f: A \rightarrow A^{\prime}$ un homomorphisme d'anneaux, $M$ un $A$-module, $M^{\prime}$ un $A^{\prime}$-module quotient de $A^{\prime} \otimes_{A} M$ et $u: M \rightarrow A^{\prime} \otimes_{A} M \rightarrow M^{\prime}$ l'application composée. Si I est un idéal de $A$ tel que $f(I)$ soit un idéal de $A^{\prime}$, alors $f(I) M^{\prime}=u(I M)$. Ainsi, l'image par $u$ du A-module IM est un $A^{\prime}$-module.

Tout élément de $M^{\prime}$ est somme finie d'éléments de la forme $a^{\prime} u(x)$ avec $a^{\prime} \in A^{\prime}$ et $x \in M$ puisque $M^{\prime}$ est un quotient de $A^{\prime} \otimes_{A} M$; comme $f(I)$ est un idéal de $A^{\prime}$, les éléments de $f(I) M^{\prime}$ sont ceux qu'on peut écrire comme somme finie d'éléments de la forme $a^{\prime} u(x)$ avec $a^{\prime} \in f(I)$ et $x \in M$; or, si $a^{\prime}=f(a)$ avec $a \in I$, on a $a^{\prime} u(x)=u(a x)$.

Remarque 1.5. - Gardant les notations de 1.4, il ne faut pas croire que $I M$ soit un $A^{\prime}$-module lorsque $u$ n'est pas injectif. Par exemple, si $A$ est un anneau intègre et si $A^{\prime}$ est une $A$-algèbre finie contenue dans le corps des fractions de $A$ et distincte de $A$, alors le conducteur $I$ de $A \rightarrow A^{\prime}$ est non nul et pour tout 
élément non nul $t \in I$, les inclusions $t A \subset t A^{\prime} \subset A$ sont strictes; en particulier, $t A$ n'est pas un $A^{\prime}$-module. Prenant $M=A / t A$, on a donc $I M=I / t A$, et $I M$ n'est pas, lui non plus, un $A^{\prime}$-module.

1.6. Pour clore ces généralités, il faut rappeler ce qu'est un produit fibré de catégories ( $c f .[3]$ p. 358).

Étant donnés deux foncteurs $G: \mathcal{B} \rightarrow \mathcal{B}^{\prime}$ et $P^{\prime}: \mathcal{A}^{\prime} \rightarrow \mathcal{B}^{\prime}$, on appelle produit fibré de $\mathcal{B}$ et de $\mathcal{A}^{\prime}$ au-dessus de $\mathcal{B}^{\prime}$ et on note $\mathcal{B} \times \mathcal{B}^{\prime} \mathcal{A}^{\prime}$, la catégorie dont les objets sont les triplets $\left(N, s, M^{\prime}\right)$ où $N$ est un objet de $\mathcal{B}, M^{\prime}$ un objet de $\mathcal{A}^{\prime}$ et $s$ un isomorphisme de $\mathcal{B}^{\prime}$

$$
s: G(N) \stackrel{\sim}{\longrightarrow} P^{\prime}\left(M^{\prime}\right),
$$

et dont les flèches de l'objet $\left(N_{0}, s_{0}, M_{0}^{\prime}\right)$ vers l'objet $\left(N_{1}, s_{1}, M_{1}^{\prime}\right)$ sont les couples $\left(b, a^{\prime}\right)$ où $b: N_{0} \rightarrow N_{1}$ est une flèche de $\mathcal{B}, a^{\prime}: M_{0}^{\prime} \rightarrow M_{1}^{\prime}$ une flèche de $\mathcal{A}^{\prime}$, tels que le diagramme suivant soit commutatif :

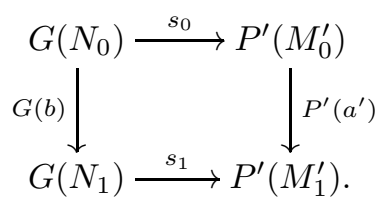

On a donc deux foncteurs $F: \mathcal{B} \times{ }_{\mathcal{B}^{\prime}} \mathcal{A}^{\prime} \rightarrow \mathcal{A}^{\prime}$ et $P: \mathcal{B} \times{ }_{\mathcal{B}^{\prime}} \mathcal{A}^{\prime} \rightarrow \mathcal{B}$ et un isomorphisme de foncteurs

$$
\sigma: G P \stackrel{\sim}{\longrightarrow} P^{\prime} F
$$

Cette construction résout le «problème universel » suivant : étant donnés deux foncteurs $H: \mathcal{A} \rightarrow \mathcal{A}^{\prime}$ et $Q: \mathcal{A} \rightarrow \mathcal{B}$, et un isomorphisme fonctoriel $\tau: G Q \rightarrow P^{\prime} H$, il existe un unique foncteur $T: \mathcal{A} \rightarrow \mathcal{B} \times_{\mathcal{B}^{\prime}} \mathcal{A}^{\prime}$ tel que $H=F T$, $Q=P T$ et $\tau=\sigma * T$.

Si les catégories et les foncteurs donnés sont additifs, il en est de même du produit fibré.

\section{Modules sur un produit fibré d'anneaux}

2.1. Soient $g: B \rightarrow B^{\prime}$ et $p^{\prime}: A^{\prime} \rightarrow B^{\prime}$ deux homomorphismes d'anneaux; il s'agit ici de relier les catégories $\operatorname{Mod}\left(B \times{ }_{B^{\prime}} A^{\prime}\right)$ et $\operatorname{Mod}(B) \times_{\operatorname{Mod}\left(B^{\prime}\right)} \operatorname{Mod}\left(A^{\prime}\right)$, ce produit fibré de catégories étant relatif aux foncteurs extension des scalaires :

$$
g^{\star}: \operatorname{Mod}(B) \longrightarrow \operatorname{Mod}\left(B^{\prime}\right) \text { et } p^{\prime \star}: \operatorname{Mod}\left(A^{\prime}\right) \longrightarrow \operatorname{Mod}\left(B^{\prime}\right) .
$$


Posons $A=B \times_{B^{\prime}} A^{\prime}$, de sorte qu'on a le carré cartésien

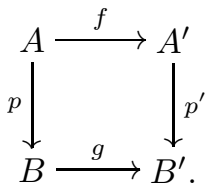

Comme $g p=p^{\prime} f$, on a un isomorphisme de foncteurs

$$
\sigma: g^{*} p^{*} \stackrel{\sim}{\longrightarrow} p^{\prime *} f^{*} .
$$

D'après la propriété universelle du produit fibré de catégories, on en déduit un foncteur covariant additif

$$
\begin{aligned}
T: \operatorname{Mod}\left(B \times_{B^{\prime}} A^{\prime}\right) & \longrightarrow \operatorname{Mod}(B) \times_{\operatorname{Mod}\left(B^{\prime}\right)} \operatorname{Mod}\left(A^{\prime}\right), \\
M & \longmapsto T(M)=\left(M \otimes_{A} B, \sigma_{M}, M \otimes_{A} A^{\prime}\right),
\end{aligned}
$$

où $\sigma_{M}$ est l'isomorphisme $g^{*} p^{*}(M) \stackrel{\sim}{\rightarrow} p^{\prime *} f^{*}(M)$. Ce foncteur possède un adjoint à droite $S$, défini par

$$
S\left(N, s, M^{\prime}\right)=\left\{\left(y, x^{\prime}\right) \in N \times M^{\prime} \text { tels que } s(1 \otimes y)=1 \otimes x^{\prime}\right\} .
$$

Le carré suivant est donc cartésien :

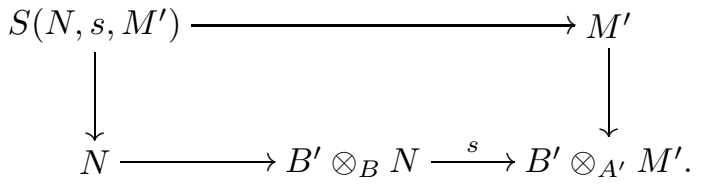

ThÉORÈme 2.2. - Soient $g: B \rightarrow B^{\prime}$ et $p^{\prime}: A^{\prime} \rightarrow B^{\prime}$ deux homomorphismes d'anneaux et $T, S$ le couple de foncteurs adjoints définis ci-dessus :

$$
\operatorname{Mod}\left(B \times_{B^{\prime}} A^{\prime}\right) \stackrel{S}{\stackrel{S}{\leftrightarrows}} \operatorname{Mod}(B) \times_{\operatorname{Mod}\left(B^{\prime}\right)} \operatorname{Mod}\left(A^{\prime}\right) .
$$

Posons $A=B \times{ }_{B^{\prime}} A^{\prime}$, de sorte qu'on a le carré cartésien

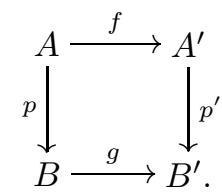

Supposons que $p^{\prime}$ soit surjectif. Alors :

i) Le morphisme d'adjonction $T S \rightarrow$ Id est un isomorphisme.

ii) Un A-module $M$ est nul si et seulement si $T(M)=0$.

iii) Pour tout A-module $M$, l'application d'adjonction $M \rightarrow S T(M)$ est surjective, son noyau est annulé par $I=\operatorname{Ker}(p)$ et est contenu dans $I M$.

BULLETIN DE LA SOCIÉTÉ MATHÉMATIQUE DE FRANCE 
En particulier, pour toute A-algèbre $A \rightarrow \bar{A}$, l'homomorphisme

$$
\bar{A} \longrightarrow \bar{A} \otimes_{A} A^{\prime} \times_{\bar{A} \otimes_{A} B^{\prime}} \bar{A} \otimes_{A} B
$$

est surjectif et son noyau est de carré nul.

iv) Désignons, pour tout anneau $R, \operatorname{par} \mathcal{C}(R)$ la catégorie des $R$-modules de type fini (resp. plats, plats et de type fini, projectifs de type fini). Alors $S$ définit par restriction un foncteur

$$
S_{\mathcal{C}}: \mathcal{C}(B) \times_{\mathcal{C}\left(B^{\prime}\right)} \mathcal{C}\left(A^{\prime}\right) \longrightarrow \mathcal{C}\left(B \times{ }_{B^{\prime}} A^{\prime}\right)
$$

qui est une équivalence de catégories dans les cas respés. En particulier, pour qu'un $A$-module $P$ soit plat (resp. etc.) il faut et il suffit que $A^{\prime} \otimes_{A} P$ soit un $A^{\prime}$-module plat et que $B \otimes_{A} P$ soit un $B$-module plat (resp. etc).

Remarques 2.3. - a) L'application surjective $M \rightarrow S T(M)$ évoquée en iii) n'est pas nécessairement un isomorphisme comme le montre le module $M=A / t A$ de 1.5 , pour lequel cette application s'identifie à la surjection (non injective) $A / t A \rightarrow A / t A^{\prime}$.

b) La partie de l'assertion iv) relative aux modules projectifs de type fini est due à Milnor, [12] p. 20 ou [3] p. 479; celle relative aux modules plats est reprise de [8].

Démonstration du théorème 2.2. - Posons $I=\operatorname{Ker}(p)$ et $I^{\prime}=\operatorname{Ker}\left(p^{\prime}\right)$, de sorte que $f$ induit un isomorphisme de $I$ sur $I^{\prime}(1.3)$.

i) Soit $\left(N, s, M^{\prime}\right)$ un objet de la catégorie produit; posons $M=S\left(N, s, M^{\prime}\right)$ et $N^{\prime}=B^{\prime} \otimes_{A^{\prime}} M^{\prime}$; on a donc le carré cartésien suivant (cf. (2.1.2))

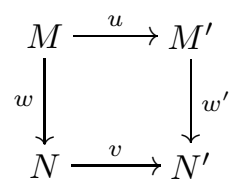

où $w^{\prime}$ est l'application canonique $M^{\prime} \rightarrow B^{\prime} \otimes_{A^{\prime}} M^{\prime}$ et où $v$ est le composé

$$
N \stackrel{\text { can. }}{\longrightarrow} B^{\prime} \otimes_{B} N \stackrel{s}{\longrightarrow} B^{\prime} \otimes_{A^{\prime}} M^{\prime}=N^{\prime} .
$$

Il faut montrer que les applications canoniques

$$
\bar{u}: A^{\prime} \otimes_{A} M \longrightarrow M^{\prime} \quad \text { et } \bar{w}: B \otimes_{A} M \longrightarrow N,
$$

déduites de $u$ et de $w$ respectivement, sont des isomorphismes.

Notons d'abord que l'application $\bar{v}: A^{\prime} \otimes_{A} N \rightarrow N^{\prime}$ est un isomorphisme : en effet, d'après (1.3), $B^{\prime}$ est isomorphe à $A^{\prime} \otimes_{A} B$; comme $N$ est un $B$-module, $\bar{v}$ est l'application composée

$$
A^{\prime} \otimes_{A} N \stackrel{\sim}{\longrightarrow} A^{\prime} \otimes_{A} B \otimes_{B} N \stackrel{\sim}{\longrightarrow} B^{\prime} \otimes_{B} N \underset{s}{\stackrel{\sim}{\longrightarrow}} N^{\prime} .
$$

C'est donc un isomorphisme. 
Notons ensuite que la surjectivité de $p^{\prime}$ entraîne celle de $w^{\prime}=p^{\prime} \otimes 1_{M^{\prime}}$, donc celle de $w$ puisque le carré (2.3.1) est cartésien; par suite, $\bar{w}$ est surjectif, ainsi que l'application $1 \otimes w$ du diagramme suivant déduit de (2.3.1) :

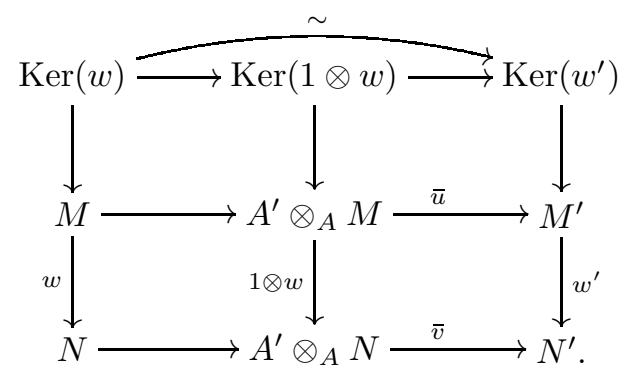

Comme $u$ induit un isomorphisme de $\operatorname{Ker}(w)$ sur $\operatorname{Ker}\left(w^{\prime}\right)$, le lemme du serpent appliqué à la partie droite de ce diagramme montre que l'application $\bar{u}$ est surjective. Le lemme 1.4 implique alors que l'on a $u(I M)=I^{\prime} M^{\prime}=\operatorname{Ker}\left(w^{\prime}\right)$; mais $I M$ est un sous-module de $\operatorname{Ker}(w)$ et $u$ induit un isomorphisme de $\operatorname{Ker}(w)$ sur $\operatorname{Ker}\left(w^{\prime}\right)=I^{\prime} M^{\prime}$; on a donc

$$
\operatorname{Ker}(w)=I M .
$$

Cela implique que $\bar{w}$ est injectif (donc est un isomorphisme) puisque $B$ est isomorphe à $A / I$.

Il reste donc à montrer que l'application $\bar{u}$ est injective, ou encore, comme on le voit sur le diagramme précédent, que $\operatorname{Ker}(1 \otimes w) \longrightarrow \operatorname{Ker}\left(w^{\prime}\right)$ est injective; pour cela, en utilisant de nouveau le fait que $u$ établit un isomorphisme $\operatorname{Ker}(w) \stackrel{\sim}{\rightarrow} \operatorname{Ker}\left(w^{\prime}\right)$, il suffit de montrer que $\operatorname{Ker}(w) \rightarrow \operatorname{Ker}(1 \otimes w)$ est surjective ; or, $\operatorname{Ker}(1 \otimes w)$ est l'image de l'application $A^{\prime} \otimes \operatorname{Ker}(w)=A^{\prime} \otimes I M \rightarrow A^{\prime} \otimes M$ et $A^{\prime} f(I)=f(I)$.

Cela achève la démonstration de l'assertion i).

ii) Soit $M$ un $A$-module tel que $B \otimes_{A} M=M / I M=0$ et $A^{\prime} \otimes_{A} M=0$; il faut montrer que $M=0$. La première hypothèse implique que l'application canonique $I \otimes_{A} M \rightarrow M$ est surjective; or, comme $f$ induit un isomorphisme $A$-linéaire de $I$ sur le $A^{\prime}$-module $f(I)$, on a des isomorphismes $I \otimes_{A} M \simeq$ $f(I) \otimes_{A^{\prime}}\left(A^{\prime} \otimes_{A} M\right) \simeq 0$.

iii) Soit $M$ un $A$-module. Pour prouver que l'application $\alpha: M \rightarrow S T(M)$ est surjective, il suffit, en utilisant l'exactitude à droite de $T$ et la partie ii), de prouver que $T(\alpha): T(M) \rightarrow T S T(M)$ est surjective. Or, c'est même un isomorphisme puisque le morphisme composé $T \rightarrow T S T \rightarrow T$ est, par adjonction, le morphisme identique et que le morphisme $T S \rightarrow$ Id est un isomorphisme d'après i). Mais on peut aussi voir cela par un procédé différent et qui donne prise sur le noyau $M_{0}=\operatorname{Ker}(\alpha)$. En effet, par définition du produit cartésien, 
on a une suite exacte de $A$-modules

$$
0 \rightarrow A \longrightarrow A^{\prime} \times B \stackrel{q}{\longrightarrow} B^{\prime} \rightarrow 0
$$

où $q$ est l'application définie par $q\left(a^{\prime}, b\right)=p^{\prime}\left(a^{\prime}\right)-g(b)$. On en tire la suite exacte

$$
\operatorname{Tor}_{1}^{A}\left(M, B^{\prime}\right) \longrightarrow M \longrightarrow A^{\prime} \otimes_{A} M \times B \otimes_{A} M \stackrel{q \otimes 1}{\longrightarrow} B^{\prime} \otimes_{A} M \rightarrow 0,
$$

et on a $S T(M)=\operatorname{Ker}(q \otimes 1)$; cela montre de nouveau que l'application $\alpha$ : $M \rightarrow S T(M)$ est surjective. L'exactitude montre aussi que le noyau $M_{0}$ de $\alpha$ est égal à l'image de $\operatorname{Tor}_{1}^{A}\left(M, B^{\prime}\right) \rightarrow M$; comme $B^{\prime}$ est annulé par $I$, il en est de même de $M_{0}$; enfin, comme $B$ est isomorphe à $A / I$, on voit que $M_{0}$ est contenu dans $I M$.

iv) Soit $\left(N, s, M^{\prime}\right)$ un triplet objet de la catégorie produit fibré tel que les modules $N$ et $M^{\prime}$ vérifient l'une des propriétés de l'énoncé; il faut montrer que $M=S\left(N, s, M^{\prime}\right)$ la vérifie aussi. Utilisant i), on voit qu'il suffit de montrer que si $M$ est un $A$-module tel que $B \otimes_{A} M$ et $A^{\prime} \otimes_{A} M$ aient l'une des propriétés envisagées, il en est de même de $M$. Notons qu'on peut remplacer $A^{\prime}$ par $A^{\prime} \times B$ et $B^{\prime}$ par $B^{\prime} \times B$ puisque l'un des carrés

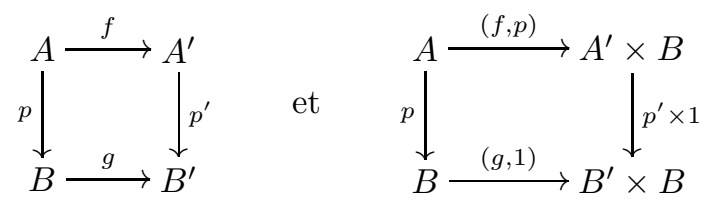

est cartésien si et seulement si l'autre l'est; on peut donc supposer que $g$, et par suite $f$, sont injectifs.

Considérons la platitude : montrons d'abord que $\operatorname{Tor}_{1}^{A}(M, A / I)=0$, c'està-dire que $M \otimes_{A} I \rightarrow M$ est injectif; comme $I$ est un idéal de $A^{\prime}$, la flèche verticale de gauche du diagramme commutatif

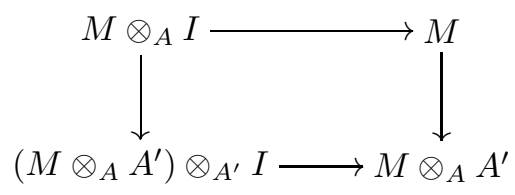

est un isomorphisme; d'autre part, comme $M \otimes_{A} A^{\prime}$ est un $A^{\prime}$-module plat, la flèche du bas est injective, donc aussi celle du haut.

En utilisant [5], AC I, p. 57, prop. 2, on en déduit que pout tout $A$-module $N$ annulé par $I$, on a $\operatorname{Tor}_{1}^{A}(M, N)=0$. Soient a un idéal de $A$ et $N=\mathfrak{a} A^{\prime} / \mathfrak{a}$, de sorte qu'on a la suite exacte

$$
0 \rightarrow \mathfrak{a} \longrightarrow \mathfrak{a} A^{\prime} \longrightarrow N \rightarrow 0
$$

Comme $I$ est un idéal de $A^{\prime}$, on $I A^{\prime}=I$ et par suite, $I N=0$; en appliquant ce qui précède, on voit que l'application $M \otimes_{A} \mathfrak{a} \rightarrow M \otimes_{A} \mathfrak{a} A^{\prime}$ est injective; d'autre 
part, comme $M \otimes_{A} A^{\prime}$ est un $A^{\prime}$-module plat, l'application $M \otimes_{A} \mathfrak{a} A^{\prime} \rightarrow M \otimes_{A} A^{\prime}$ est injective; on en déduit immédiatement que $M \otimes_{A} \mathfrak{a} \rightarrow M$ est injective, donc que $M$ est un $A$-module plat.

Considérons la propriété d'être de type fini : en relevant des générateurs, on peut construire un sous-module de type fini $M_{0}$ de $M$ tel que $T\left(M / M_{0}\right)=0$, il suffit donc d'appliquer ii).

Il reste à envisager le cas des modules projectifs de type fini, c'est-à-dire plats et de présentation finie; soit donc $M$ un $A$-module tel que les composants de $T(M)$ soient plats et de présentation finie; d'après ce qui précède, on voit déjà que $M$ est plat et de type fini; on peut donc construire une suite exacte $0 \rightarrow N \rightarrow L \rightarrow M \rightarrow 0$ où $L$ est un $A$-module libre de type fini et il faut montrer que $N$ est de type fini; or, comme $M$ est plat, on a la suite exacte (on oublie les isomorphismes $\sigma$ )

$$
0 \rightarrow T(N) \longrightarrow T(L) \longrightarrow T(M) \rightarrow 0 .
$$

Cela montre que les composants de $T(N)$ sont des modules de type fini, donc que $N$ est de type fini d'après les cas déjà envisagés.

\section{Traduction dans le langage de la théorie de la descente}

3.1. Rappelons d'abord quelques définitions introduites par Grothendieck dans [10].

Soit $f: A \rightarrow A^{\prime}$ un homomorphisme injectif d'anneaux; une donnée de descente sur un $A^{\prime}$-module $M^{\prime}$ est un isomorphisme

$$
\varphi: M^{\prime} \otimes_{A} A^{\prime} \longrightarrow A^{\prime} \otimes_{A} M^{\prime}
$$

qui est $A^{\prime} \otimes_{A} A^{\prime}$-linéaire et qui satisfait la condition de cocycle

$$
\varphi_{1}=\varphi_{0} \varphi_{2}
$$

où l'isomorphisme $\varphi_{i}$ est déduit de $\varphi$ par l'extension des scalaires relative au morphisme $A^{\prime} \otimes_{A} A^{\prime} \rightarrow A^{\prime} \otimes_{A} A^{\prime} \otimes_{A} A^{\prime}$ qui insère 1 à la $i$-ème place.

Désignons par $u_{0}$ et $u_{1}$ les applications $M^{\prime} \rightarrow A^{\prime} \otimes_{A} M^{\prime}$ définies par

$$
u_{0}\left(x^{\prime}\right)=1 \otimes x^{\prime} \quad \text { et } \quad u_{1}\left(x^{\prime}\right)=\varphi\left(x^{\prime} \otimes 1\right) .
$$

En introduisant le noyau de cette double flèche

$$
M=\operatorname{Ker}\left(u_{0}, u_{1}\right)=\left\{x^{\prime} \in M^{\prime} \mid 1 \otimes x^{\prime}=\varphi\left(x^{\prime} \otimes 1\right)\right\},
$$

on obtient la suite exacte (au sens de [10] p. 301)

$$
M \stackrel{u}{\longrightarrow} M^{\prime} \underset{u_{1}}{\stackrel{u_{0}}{\longrightarrow}} A^{\prime} \otimes_{A} M^{\prime} .
$$

Soit $m: A^{\prime} \otimes_{A} M^{\prime} \rightarrow M^{\prime}$ l'application définie par $m\left(a^{\prime} \otimes x^{\prime}\right)=a^{\prime} x^{\prime}$; alors on a

$$
m u_{0}=m u_{1}=\operatorname{id}_{M^{\prime}} .
$$


(Il suffit de remarquer que le changement de base associé à l'homomorphisme $A^{\prime} \otimes_{A} A^{\prime} \rightarrow A^{\prime}$ donné par la multiplication, transforme la donnée de descente $\varphi$ en l'identité de $M^{\prime}$, en vertu de la condition de cocycle.)

Finalement, on dit que la donnée de descente $\varphi$ est effective si l'application $A^{\prime}$-linéaire

$$
\bar{u}: A^{\prime} \otimes_{A} M \longrightarrow M^{\prime}
$$

déduite de l'injection $u$ est un isomorphisme.

THÉORÈME 3.2. - Une donnée de descente est effective si elle le devient après passage au quotient par le conducteur. Plus précisement, soit I le conducteur d'un homomorphisme injectif $f: A \rightarrow A^{\prime}$; désignons par

$$
g: B=A / I \longrightarrow B^{\prime}=A^{\prime} / f(I),
$$

l'homomorphisme déduit de $f$ par passage aux quotients. Soit $M^{\prime}$ un $A^{\prime}$-module muni d'une donnée de descente $\varphi$; posons $N^{\prime}=M^{\prime} / f(I) M^{\prime}$. Si la donnée de descente

$$
\bar{\varphi}: N^{\prime} \otimes_{B} B^{\prime} \longrightarrow B^{\prime} \otimes_{B} N^{\prime}
$$

est effective, alors $\varphi$ est effective.

3.3. Montrons que ce théorème est une conséquence du théorème 2.2. Reprenons les notations introduites en 3.1, désignons par $v_{1}, v_{0}$ les applications de $N^{\prime}$ dans $B^{\prime} \otimes_{B} N^{\prime}$ déduites de $u_{1}$ et $u_{0}$ par passage aux quotients et notons $v: N \rightarrow N^{\prime}$ le noyau de $\left(v_{0}, v_{1}\right)$, de sorte que dans le diagramme commutatif suivant, en oubliant $m$, les deux lignes sont exactes.

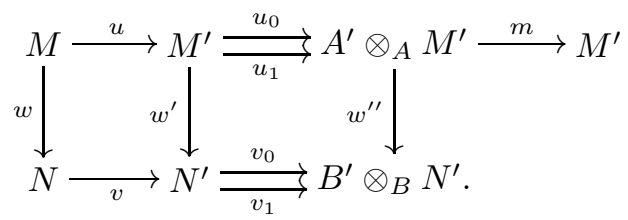

Par hypothèse, la donnée de descente sur $N^{\prime}$ est effective, autrement dit, l'application $\bar{v}: B^{\prime} \otimes_{B} N \rightarrow N^{\prime}$ déduite de l'injection $v$ est un isomorphisme et il faut en déduire la propriété analogue pour $u$; d'après 2.2 , i), il suffit de montrer que le carré de gauche est cartésien. Considérons donc un élément $x^{\prime} \in M^{\prime}$ tel que $v_{0} w^{\prime}\left(x^{\prime}\right)=v_{1} w^{\prime}\left(x^{\prime}\right)$, c'est-à-dire, les carrés étant commutatifs, tel que $w^{\prime \prime} u_{0}\left(x^{\prime}\right)=w^{\prime \prime} u_{1}\left(x^{\prime}\right)$; il faut vérifier que $u_{0}\left(x^{\prime}\right)=u_{1}\left(x^{\prime}\right)$; comme $m u_{0}=m u_{1}(3.1 .2)$, on est ramené à prouver que $\operatorname{Ker}\left(w^{\prime \prime}\right) \cap \operatorname{Ker}(m)=0$; pour cela remarquons que $B^{\prime}$ est isomorphe à $A^{\prime} \otimes_{A} B$, si bien que $w^{\prime \prime}$ se déduit de $w^{\prime}$ par « extension des scalaires » à $A^{\prime}$; par suite $\operatorname{Ker}\left(w^{\prime \prime}\right)$ est égal à l'image de $A^{\prime} \otimes_{A} f(I) M^{\prime}$ dans $A^{\prime} \otimes_{A} M^{\prime}$; mais comme $I$ est le conducteur de $f$, on voit finalement grâce à 1.4 que les éléments de $\operatorname{Ker}\left(w^{\prime \prime}\right)$ peuvent être écrits sous la forme $1 \otimes y^{\prime}$ avec $y^{\prime} \in M^{\prime}$, donc que $\operatorname{Ker}\left(w^{\prime \prime}\right) \cap \operatorname{Ker}(m)=0$. 
3.4. Ce résultat n'a d'intérêt que si toute donnée de descente relative à $A / I \rightarrow A^{\prime} / f(I)$ est effective, par exemple si cet homomorphisme est fidèlement plat, ou admet une rétraction.

L'auteur de ces lignes espérait (en 1970) que ce résultat et des procédés analogues à ceux utilisés dans [8] lui auraient permis de montrer que toute donnée de descente, sur un module plat, relative à un homomorphisme fini d'anneaux noethériens est effective, comme il est conjecturé dans [10] p. 307. J. Venken [20] a montré que cette conjecture est fausse; un contre-exemple plus frappant a été donné ensuite par Anne Philippe [15].

\section{Pincements}

4.1. Reprenons tout d'abord la construction d'une courbe pincée, construction devenue familière aux géomètres depuis le livre de Serre [19].

Soient $C^{\prime}$ une courbe propre et lisse sur un corps algébriquement clos $k$ et $x, y$ deux points (fermés) de $C^{\prime}$; Serre donne (loc. cit., p. 68 et suivantes) la construction d'une courbe $C$ propre et intègre sur $k$ et d'un morphisme fini $f: C^{\prime} \rightarrow C$ tels que $x$ et $y$ aient la même image $z$ par $f$ et que $f$ induise un isomorphisme de l'ouvert $C^{\prime}-\{x, y\}$ sur l'ouvert $C-\{z\}$; on peut donc considérer que la courbe $C$ est obtenue à partir de $C^{\prime}$ en «identifiant» les points $x$ et $y$. Plus généralement (loc. cit.), si on se donne un «module» $\operatorname{sur} C^{\prime}$ (on dirait, aujourd'hui, un cycle 1-codimensionnel positif), il existe une courbe $C$ munie d'un point $z$ et un morphisme fini $f: C^{\prime} \rightarrow C$ tels que l'ensemble $f^{-1}(z)$ soit égal au support $S$ du module et tels que $f$ induise un isomorphisme de l'ouvert $C^{\prime}-S$ sur l'ouvert $C-\{z\}$; on peut dire alors que $C$ est obtenue en « pinçant» $C^{\prime}$ en $S$.

On se propose d'étendre aux schémas ce procédé de construction.

Explicitons d'abord la situation précédente en des termes plus naturellement généralisables : soit $D \subset C^{\prime}$ le sous-schéma fermé associé au «module » considéré ( $c f$. [9], EGA IV, 21.7.1); il est muni canoniquement d'un morphisme fini $g: D \rightarrow \operatorname{Spec}(k)$; d'autre part, la donnée du point $z$ de $C$ est équivalente à la donnée d'une $k$-immersion fermée $\operatorname{Spec}(k) \rightarrow C$; d'après la construction donnée dans (loc. cit.) et le critère 4.3 ci-dessous, le carré de morphismes de schémas

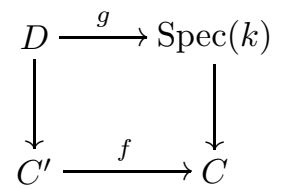

est co-cartésien dans la catégorie des espaces annelés, c'est-à-dire qu'il fait de $C$ la somme (dans la catégorie en question) de $C^{\prime}$ et de $\operatorname{Spec}(k)$ amalgamée en $D$.

D'autre part, la formule (9) de la page 70 de loc. cit. se traduit par le fait que le carré ci-dessus est aussi cartésien.

BULlETIN DE LA SOCIÉTÉ MATHÉMATIQUE DE FRANCE 
4.2. Par analogie avec ce qui précède, on peut énoncer le problème du pincement pour des schémas dans les termes suivants.

Soient $Y^{\prime}$ un sous-schéma fermé d'un schéma $X^{\prime}$ et $g: Y^{\prime} \rightarrow Y$ un morphisme de schémas; on sait que l'espace annelé somme amalgamée $X=X^{\prime} \sqcup_{Y^{\prime}} Y$ existe toujours (l'énoncé 4.3 en donne une description explicite), de sorte qu'on a un carré cocartésien de morphismes d'espaces annelés :

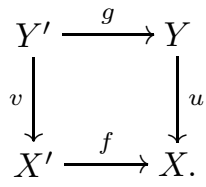

On cherche des conditions pour que :

a) $X$ soit un schéma;

b) le carré soit cartésien, c'est-à-dire que le morphisme $Y^{\prime} \rightarrow X^{\prime} \times_{X} Y$ soit un isomorphisme.

Dans la suite nous supposerons toujours que $g$ est affine, laissant de côté le problème des «contractions » où $g$ est supposé propre ( $c f$. le critère de Castelnuovo); il est alors naturel de vouloir en outre que

c) $f$ soit affine.

(Dans 7.3, on construit un carré cocartésien comme ci-dessus, où $X$ est un schéma, où $X, Y$ et $Y^{\prime}$ sont affines, et où $X^{\prime}$ n'est pas affine ; cet exemple montre que la condition c) n'est pas conséquence des autres.)

Notons que lorsque le morphisme $g$ est lui aussi une immersion fermée, l'existence du schéma $X$ était déjà connue, et que cette cette situation est traitée en détail dans la thèse d'Anantharaman [1].

SCOLIE 4.3. - Considérons un carré commutatif de morphismes d'espaces annelés:

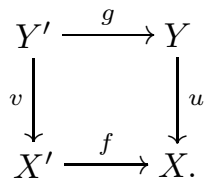

a) Pour que ce carré soit cocartésien, il faut et il suffit que les trois propriétés suivantes soient vérifiées :

i) Le carré des applications des ensembles sous-jacents est cocartésien, ce qui veut dire que $X=f\left(X^{\prime}\right) \cup u(Y)$ et que pour tout couple $x^{\prime}, y$ de points de $X^{\prime}$ et $Y$, on a $f\left(x^{\prime}\right)=u(y)$ si et seulement si il existe $y^{\prime} \in Y^{\prime}$ tel que $v\left(y^{\prime}\right)=x^{\prime}$ et $g\left(y^{\prime}\right)=y$.

ii) L'application continue $X^{\prime} \sqcup Y \rightarrow X$, déduite de $f$ et $u$, est submersive, ce qui veut dire qu'elle est surjective et qu'une partie $U$ de $X$ est ouverte dès que $u^{-1}(U)$ et $f^{-1}(U)$ sont ouverts. 
iii) Notant $h=u g=f v$, le carré d'homomorphismes de faisceaux d'anneaux sur $X$

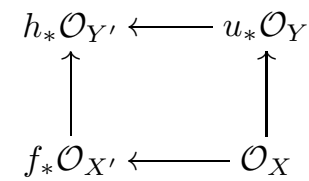

est cartésien.

b) Supposons que l'application induite par $v$ sur les ensembles sous-jacents soit injective; alors la propriété i) est équivalente à :

$\left.i^{\prime}\right) u$ est injectif et $f$ induit une bijection de $X^{\prime}-v\left(Y^{\prime}\right)$ sur $X-u(Y)$.

c) Supposons que $v$ induise un homéomorphisme de $Y^{\prime}$ sur une partie fermée de $X^{\prime}$ et que le carré ci-dessus soit cocartésien. Alors u induit un homéomorphisme de $Y$ sur une partie fermée de $X$ et $f$ définit un isomorphisme de l'espace annelé induit par $X^{\prime}$ sur $X^{\prime}-v\left(Y^{\prime}\right)$ sur l'espace annelé induit par $X$ sur $X-u(Y)$.

LEMme 4.4. - Supposons que le carré commutatif de morphismes de schémas

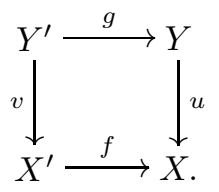

soit cocartésien dans la catégorie des espaces annelés. Supposons de plus que les quatre morphismes soient quasi-compacts et quasi-séparés et que le morphisme $X^{\prime} \sqcup Y \rightarrow X$ déduit de $u$ et de $f$ soit universellement submersif. Alors, le carré obtenu par un changement de base plat $\bar{X} \rightarrow X$ est encore cocartésien dans la catégorie des espaces annelés.

On utilise le critère 4.3 , a) : la vérification de la propriété i) est immédiate si on tient compte du fait suivant : si $Z \rightarrow X$ et $\bar{X} \rightarrow X$ sont deux morphismes de schémas, l'application canonique

$$
\left(Z \times_{X} \bar{X}\right)_{\mathrm{ens}} \longrightarrow Z_{\mathrm{ens}} \times_{X_{\mathrm{ens}}} \bar{X}_{\mathrm{ens}}
$$

est surjective; la propriété ii) figure dans les hypothèses; pour iii), on utilise d'une part le fait que le produit tensoriel par un module plat préserve l'exactitude des diagrammes, et d'autre part, le fait que la formation de l'image directe par un morphisme quasi-compact et quasi-séparé commute aux changements de base plats [9], EGA IV, 2.3.1).

\section{Existence de pincements ; les cas utiles}

Commençons par le cas affine.

BULlETiN DE LA SOCIÉtÉ MATHÉMATIQUE DE FRANCE 
THÉORÈme 5.1. - Soit

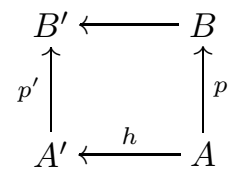

un carré cartésien d'homomorphismes d'anneaux où $p^{\prime}$ est surjectif. Alors le carré

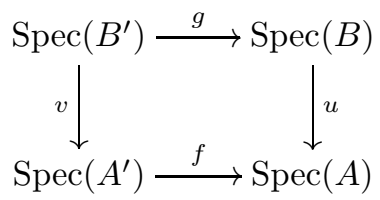

est co-cartésien dans la catégorie des espaces annelés.

Rappelons que l'hypothèse signifie que $h$ et $p$ permettent d'identifier $A$ au produit fibré d'anneaux $A^{\prime} \times_{B^{\prime}} B$ et qu'elle implique d'après 1.3 , que :

a) $p$ est surjectif;

b) l'homomorphisme $h$ induit un isomorphisme de $I=\operatorname{Ker}(p)$ sur $\operatorname{Ker}\left(p^{\prime}\right)$;

c) le carré est cocartésien, i.e. l'homomorphisme $B \otimes_{A} A^{\prime} \rightarrow B^{\prime}$ est un isomorphisme, ou encore, compte-tenu de a) et de b), on a un isomorphisme $B^{\prime} \simeq A^{\prime} / h(I)$.

Notons d'abord que pour $t \in I, h$ induit un isomorphisme

$$
A_{t} \stackrel{\sim}{\longrightarrow} A_{h(t)}^{\prime} \text {. }
$$

En effet, cet homomorphisme est injectif car si un élément $a \in A$ vérifie la relation $h\left(a t^{m}\right)=0$, avec $m \geq 0$, alors on a $a t^{m+1} \in I \cap \operatorname{Ker}(h)$ et cette intersection est nulle d'après b); l'image de $a$ dans $A_{t}$ est donc nulle. Cet homomorphisme est aussi surjectif car, pour $a^{\prime} \in A^{\prime}$ il existe, par définition du conducteur, un élément $a \in A$ tel que $h(t) a^{\prime}=h(a)$; par suite, pour tout $n \geq 0$, on a

$$
a^{\prime} / h\left(t^{n}\right)=h(t) a^{\prime} / h\left(t^{n+1}\right)=h(a) / h\left(t^{n+1}\right) ;
$$

c'est un élément de l'image.

Posons $Y^{\prime}=\operatorname{Spec}\left(B^{\prime}\right), Y=\operatorname{Spec}(B), X^{\prime}=\operatorname{Spec}\left(A^{\prime}\right)$ et $X=\operatorname{Spec}(A)$. D'après ce qui précède, $f$ définit un isomorphisme de $X^{\prime}-Y^{\prime}=f^{-1}(X-Y)$ sur $X-Y$; comme $u$ est injectif (c'est une immersion fermée d'après a)), on voit que le carré (5.1.1) est cocartésien dans la catégorie des ensembles, puisque la condition $\mathrm{i}^{\prime}$ ) de $4.3, \mathrm{~b}$ ) est satisfaite; la condition iii) l'est aussi par définition d'un carré cartésien d'anneaux. Il reste donc à vérifier que le morphisme $X^{\prime} \sqcup Y \rightarrow X$ est submersif ${ }^{(2)}$.

$\overline{{ }^{(2)} \text { Cette vérification manque dans [3] p. } 488 .}$ 
Pour cela considérons une partie $Z$ de $X$ telle que $f^{-1}(Z)$ soit un fermé de $X^{\prime}$ et que $Z \cap Y$ soit un fermé de $Y$; il faut montrer que $Z$ est fermé. Comme $Y$ est fermé dans $X, Z \cap Y$ est fermé aussi dans $X$; par suite, on a $\bar{Z}=\overline{Z-Z \cap Y} \cup Z \cap Y$ et il suffit de montrer que $Z-Z \cap Y$ est fermé dans l'ouvert $X-Z \cap Y$, ou encore qu'on peut recouvrir cet ouvert par des ouverts $V$ tels que $V \cap Z$ soit fermé dans $V$. Choisissons, pour cela, un recouvrement de $X-Z \cap Y$ par des ouverts affine spéciaux $\operatorname{Spec}\left(A_{s}\right)$; or, « rendre $s$ inversible » dans les anneaux $A, B, A^{\prime}$ et $B^{\prime}$ préserve les hypothèses de 5.1 et permet donc d'ajouter l'hypothèse que $Z$ est contenu dans $X-Y$. Comme $f$ définit un isomorphisme de $f^{-1}(X-Y)$ sur $X-Y$ et que $f^{-1}(Z)$ est supposé fermé dans $X^{\prime}$, il suffit alors de voir que l'adhérence de $Z$ dans $X$ est contenue dans $X-Y$, ou encore, qu'il existe un ouvert $U$ de $X$ contenant $Y$ et disjoint de $Z$; c'est ce qu'affirme le lemme suivant en prenant $U^{\prime}=X^{\prime}-f^{-1}(Z)$ :

LEMme 5.2. - Sous les hypothèses et avec les notations de 5.1, pour tout ouvert $U^{\prime}$ de $X^{\prime}$ contenant $Y^{\prime}$, il existe un ouvert spécial $U=\operatorname{Spec}\left(A_{t}\right) \subset X$, contenant $Y$ et tel que $f^{-1}(U) \subset U^{\prime}$.

Soit $J^{\prime}$ un idéal de $A^{\prime}$ définissant l'ouvert $U^{\prime}$; comme le fermé $Y^{\prime}$, qui est défini par l'idéal $h(I)$, est contenu dans $U^{\prime}$, on a $h(I)+J^{\prime}=A^{\prime}$; il existe donc un élément $a \in I$ tel que $h(1-a) \in J^{\prime}$. Si on pose $t=1-a$ et $U=\operatorname{Spec}\left(A_{t}\right)$, on voit que $I+t A=A$, donc que $Y=V(I) \subset U$ et que $f^{-1}(U)=\operatorname{Spec}\left(A_{t}^{\prime}\right) \subset$ $D\left(J^{\prime}\right)=U^{\prime}$.

Remarques 5.3. - Comme $h$ induit un isomorphisme de $I$ sur l'idéal $h(I)$ de $A^{\prime}$, la multiplication par les éléments de $A^{\prime}$ définit un homomorphisme $A^{\prime} \rightarrow \operatorname{End}_{A}(I) ;$ notant $\mathfrak{a}=\operatorname{Ann}_{A}(I)$ et $\mathfrak{a}^{\prime}=\operatorname{Ann}_{A^{\prime}}(h(I))$, on a un triangle commutatif d'homomorphismes injectifs

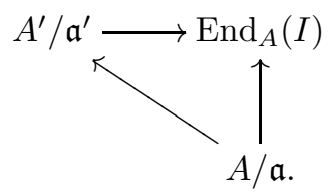

5.3.1. Supposons que l'idéal de $I$ de $A$ soit de type fini, alors la partie topologique de la démonstration de 5.1 devient triviale. En effet, tout élément de $\operatorname{End}_{A}(I)$ est alors entier sur $A$ et donc $A^{\prime} / \mathfrak{a}^{\prime}$ est entier sur $A$; comme $I \cap \mathfrak{a}$ est de carré nul, le morphisme

$$
\operatorname{Spec}\left(A^{\prime} / \mathfrak{a}^{\prime}\right) \sqcup \operatorname{Spec}(A / I) \longrightarrow \operatorname{Spec}(A)
$$

est surjectif et fermé (Cohen-Seidenberg) donc submersif; a fortiori le morphisme $\operatorname{Spec}\left(A^{\prime}\right) \sqcup \operatorname{Spec}(A / I) \rightarrow \operatorname{Spec}(A)$ est submersif. 
5.3.2. Supposons que $A$ soit noethérien et que $\mathfrak{a}^{\prime}=0$; alors $A^{\prime}$ est fini sur $A$; par suite, $A^{\prime}$ est noethérien et $g$ est un homomorphisme fini.

Réciproquement, supposons que $g$ soit fini et que $A^{\prime}$ et $B$ soient noethériens, alors $A$ est noethérien; en effet, $A^{\prime}$ est fini sur $A$, donc l'homomorphisme

$$
A \longrightarrow B \times A^{\prime}
$$

est fini et injectif, et on peut utiliser [9], EGA $0_{\mathrm{I}} 6.4 .9$.

L'énoncé suivant est moins général que 7.1 mais plus utile en géométrie.

THÉORÈme 5.4. - Soient $X^{\prime}$ un schéma, $Y^{\prime}$ un sous-schéma fermé de $X^{\prime}$ et $g: Y^{\prime} \rightarrow Y$ un morphisme fini. Considérons l'espace annelé somme amalgamée $X=X^{\prime} \sqcup_{Y^{\prime}} Y$ et le carré cocartésien

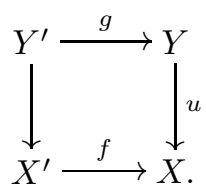

On suppose que les schémas $X^{\prime}$ et $Y$ vérifient la propriété suivante:

(AF) Tout ensemble fini de points est contenu dans un ouvert affine. Alors :

a) $X$ est un schéma vérifiant (AF);

b) le carré (5.4.1) est cartésien;

c) le morphisme $f$ est fini et u est une immersion fermée;

d) $f$ induit un isomorphisme de $X^{\prime}-Y^{\prime}$ sur $X-Y$.

L'espace annelé $\left(X, \mathcal{O}_{X}\right)$ est un schéma vérifiant la propriété $(\mathrm{AF})$ si toute partie finie $E \subset X$ est contenue dans un ouvert $U$ tel que l'espace annelé $\left(U, \mathcal{O}_{U}\right)$ induit par $X$, soit un schéma affine. Or, une partie $E$ est déterminée par sa trace $F=u^{-1}(E)$ sur $Y$ et par la partie complémentaire $E-F$, laquelle peut-être identifiée à la partie $E^{\prime}=f^{-1}(E-F)$ contenue dans $X^{\prime}-Y^{\prime}$. On va donc montrer ceci : étant données une partie finie $F \subset Y$ et une partie finie $E^{\prime} \subset X^{\prime}-Y^{\prime}$, il existe un ouvert affine $V \subset Y$ et un ouvert affine $U^{\prime} \subset X^{\prime}$ tels que

$$
F \subset V, \quad E^{\prime} \cup g^{-1}(F) \subset U^{\prime}, \quad g^{-1}(V)=U^{\prime} \cap Y^{\prime} .
$$

On pourra alors utiliser 5.1 pour voir que le schéma affine

$$
U=\operatorname{Spec}\left(\Gamma(V) \times_{\Gamma\left(U^{\prime} \cap Y^{\prime}\right)} \Gamma\left(U^{\prime}\right)\right)
$$

s'insère dans un carré cocartésien

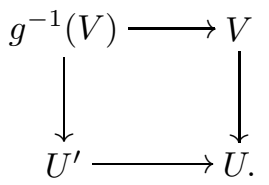


Cela permettra d'identifier $U$ à un ouvert de $X$ contenant $E$.

Considérons donc des parties finies $F$ et $E^{\prime}$ comme plus haut. La propriété (AF) entraîne déjà l'existence d'un ouvert affine $V$ de $Y$ tel que $F \subset V$, et d'un ouvert affine $U^{\prime}$ de $X^{\prime}$ contenant l'ensemble fini $E^{\prime} \cup g^{-1}(F)$ (le morphisme $g$ est fini par hypothèse); il s'agit donc de trouver des sections $s \in \Gamma\left(U^{\prime}\right)$ et $t \in \Gamma(V)$ telles que l'on ait l'égalité $g^{-1}\left(V_{t}\right)=U_{s}^{\prime} \cap Y^{\prime}$ (On note, comme d'habitude, par $V_{t}$ l'ouvert affine de $V$ où $t$ est inversible.) La construction des sections cherchées ressemble alors à celle donnée dans SGA I, p. 222-223.

On utilisera plusieurs fois le « lemme d'évitement » suivant :

LEMme 5.5. - Soient $S$ un schéma affine, $T$ un sous-schéma fermé et $Z$ une partie finie de l'ouvert $S-T$. Alors, pour toute section $t$ dans $\Gamma\left(T, \mathcal{O}_{T}\right)$, il existe une section $s \in \Gamma\left(S, \mathcal{O}_{S}\right)$, d'image $t$ et inversible aux points de $Z$; autrement dit, on a $Z \subset S_{s}$ et $S_{s} \cap T=T_{t}$. En particulier $(t=0)$, il existe une section $s$ telle que $Z \subset S_{s} \subset S-T$.

Soit $I$ le noyau de l'homomorphisme surjectif $\Gamma\left(S, \mathcal{O}_{S}\right) \longrightarrow \Gamma\left(T, \mathcal{O}_{T}\right)$ et soit $u \in \Gamma(S)$ une section d'image $t$. Si on a $Z \subset S_{u}$, on peut prendre $s=u$. Sinon, indexons les idéaux premiers de $\Gamma(S)$ associés aux points de $Z$ de sorte que

$$
u \in \mathfrak{p}_{1} \cap \cdots \cap \mathfrak{p}_{m} \quad \text { et } \quad u \notin \mathfrak{p}_{m+1} \cup \cdots \cup \mathfrak{p}_{n} .
$$

Pour $i \leq m$ l'idéal premier $\mathfrak{p}_{i}$ ne contient donc aucun des $\mathfrak{p}_{j}$ pour $j>m$; comme il ne contient pas non plus $I$ par hypothèse, il ne contient pas l'idéal $I \cap \mathfrak{p}_{m+1} \cap \cdots \cap \mathfrak{p}_{n}$. Il résulte alors de [5], AC II, p. 70, qu'il existe $a \in \Gamma(S)$ tel que

$$
a \in I \cap \mathfrak{p}_{m+1} \cap \cdots \cap \mathfrak{p}_{n} \quad \text { et } \quad a \notin \mathfrak{p}_{1} \cup \cdots \cup \mathfrak{p}_{m} .
$$

La section $s=a+u$ a les propriétés requises.

Revenons à la démonstration du théorème et considérons deux ouverts affines $V$ et $U^{\prime}$ comme plus haut; le lemme d'évitement montre d'abord qu'il existe $t \in \Gamma\left(U^{\prime} \cap Y^{\prime}\right)$ tel que

$$
g^{-1}(F) \subset\left(U^{\prime} \cap Y^{\prime}\right)_{t} \subset U^{\prime} \cap g^{-1}(V) ;
$$

appliquons maintenant le même lemme avec $S=U^{\prime}, T=U^{\prime} \cap Y^{\prime}, Z=E^{\prime}$; on en tire l'existence d'une section $s \in \Gamma\left(U^{\prime}\right)$ telle que

$$
E^{\prime} \cup g^{-1}(F) \subset U_{s}^{\prime} \quad \text { et } \quad U_{s}^{\prime} \cap Y^{\prime} \subset g^{-1}(V) .
$$

Quitte à remplacer $U^{\prime}$ par $U_{s}^{\prime}$, on peut donc supposer que $U^{\prime} \cap Y^{\prime} \subset g^{-1}(V)$. Ces données sont illustrées par le diagramme suivant :

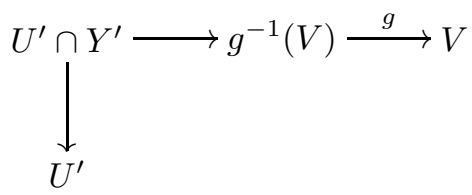

BULLETIN DE LA SOCIÉTÉ MATHÉMATIQUE DE FRANCE 
où la flèche verticale désigne une immersion fermée; on utilisera aussi le diagramme d'homomorphismes d'anneaux associé au précédent

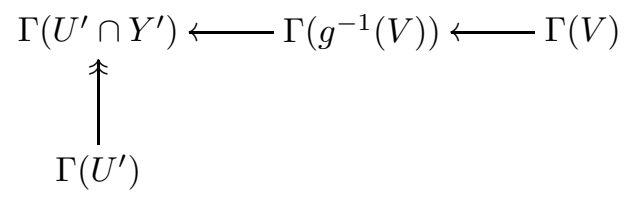

Comme $g$ est, en particulier, un morphisme fermé, l'ensemble $g\left(Y^{\prime}-Y^{\prime} \cap U^{\prime}\right)$ est un fermé de $Y$, et il est disjoint de $F$ puisque l'on a supposé $g^{-1}(F) \subset U^{\prime}$; par suite, l'ouvert

$$
\mathcal{V}=V-V \cap g\left(Y^{\prime}-Y^{\prime} \cap U^{\prime}\right)
$$

est contenu dans $V$, il contient l'ensemble fini $F$ et on a $g^{-1}(\mathcal{V}) \subset U^{\prime}$; d'après le lemme d'évitement, il existe une section $t \in \Gamma(V)$ telle que $F \subset V_{t} \subset \mathcal{V}$, donc telle que $g^{-1}\left(V_{t}\right) \subset U^{\prime} \cap Y^{\prime}$; notant $t^{\prime}$ l'image de $t$ dans $\Gamma\left(U^{\prime} \cap Y^{\prime}\right)$, on a

$$
g^{-1}\left(V_{t}\right)=\left(U^{\prime} \cap Y^{\prime}\right)_{t^{\prime}} .
$$

Une ultime application du lemme d'évitement montre qu'il existe une section $s \in \Gamma\left(U^{\prime}\right)$ relevant $t^{\prime}$ et inversible sur $E^{\prime}$.

Cela achève la démonstration de la partie a) du théorème; le fait que $f$ soit fini est un cas particulier de l'énoncé général suivant.

Proposition 5.6. - Soit

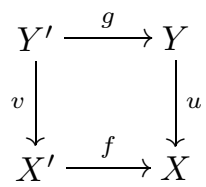

un carré cocartésien de morphismes de schémas dans lequel $f, g$ sont affines et $u, v$ des immersions fermées. Alors,

1) Le carré d'immersions fermées

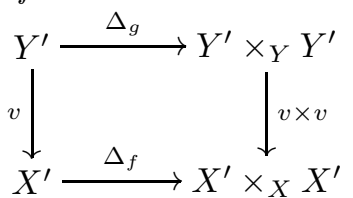

est cocartésien.

2) Si l'application $\mathcal{O}_{Y} \rightarrow g_{\star}\left(\mathcal{O}_{Y}^{\prime}\right)$ est injective, alors l'application $\mathcal{O}_{X} \rightarrow f_{\star}\left(\mathcal{O}_{X}^{\prime}\right)$ est injective. Dans ce cas, si, de plus, $X^{\prime}$ est intègre ou réduit, il en est de même de $X$.

3) Considérons pour un morphisme de schémas, la propriété d'être:
i) entier,
ii) de type fini,
iii) fini,
iv) quasi-fini,
v) net.

Alors, si g vérifie l'une de ces propriétés, $f$ la vérifie aussi. 
Toutes les propriétés sont de nature locale sur la base $X$; aussi peut-on supposer que $X$ est affine; les trois autres schémas sont alors affines et on est ramené à des énoncés relatifs à un carré cartésien d'homomorphismes d'anneaux, écrit comme en 5.1.

1) Il s'agit de vérifier que le carré suivant d'homomorphismes surjectifs est cartésien :

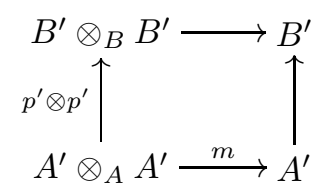

D'après le «lemme chinois », il faut montrer que $\operatorname{Ker}\left(p^{\prime} \otimes p^{\prime}\right) \cap \operatorname{Ker}(m)=0$; c'est une conséquence du fait que $I^{\prime}=\operatorname{Ker}\left(p^{\prime}\right)$ est l'image par $h: A \rightarrow A^{\prime}$ de l'idéal $I=\operatorname{Ker}(p)$; en effet, tout élément de $z \in \operatorname{Ker}\left(p^{\prime} \otimes p^{\prime}\right)$ est somme d'éléments de la forme $x \otimes y$ avec, pour chacun d'eux, $x \in I^{\prime}$ ou $y \in I^{\prime}$; or, si $x=h(a)$, avec $a \in I$, alors il existe $b \in I$ tel que $h(a) y=h(b)$, d'où

$$
x \otimes y=1 \otimes h(a) y=1 \otimes h(b)=h(b) \otimes 1 ;
$$

finalement, en faisant la somme de tels éléments, on voit que $z=1 \otimes h(c)$ pour un $c \in I$ convenable; si de plus $0=m(z)=h(c)$, alors $z=0$.

2) Cet énoncé est mis ici pour mémoire; il découle formellement de la définition d'un produit cocartésien.

3) Comme on l'a déjà rappelé, l'homomorphisme $h: A \rightarrow A^{\prime}$ induit une bijection de $I=\operatorname{Ker}(p)$ sur $I^{\prime}=\operatorname{Ker}\left(p^{\prime}\right)$; l'assertion de transfert des propriétés i) et ii) en découle immédiatement; la propriété iii) est la conjonction de i) et ii); les deux dernières sont relatives aux fibres et leur transfert de $g$ à $f$ est évident si l'on tient compte du fait que le carré est aussi cartésien et que $f$ induit un isomorphisme de $X^{\prime}-Y^{\prime}$ sur $X-Y$.

\section{Exemples de schémas propres et non projectifs}

Les résultats de cette section m'ont été signalés par Jean-Pierre Serre, en août 2000 (voir aussi [7] p. 24, note 2).

6.1. Considérons de nouveau un carré cocartésien de schémas

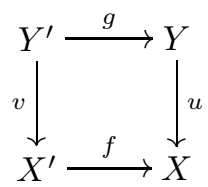

dans lequel $f$ et $g$ sont finis et $u$ et $v$ sont des immersions fermées; pour simplifier, on suppose aussi que le morphisme $\mathcal{O}_{X} \longrightarrow f_{\star}\left(\mathcal{O}_{X^{\prime}}\right)$ est injectif. Enfin, on suppose que $X^{\prime}, Y^{\prime}$ et $Y$ sont des schémas projectifs sur un corps $k$. 
Alors $X$ est propre sur $k$; en effet, comme $f: X^{\prime} \longrightarrow X$ est fini et surjectif, et que $X^{\prime}$ est séparé et universellement fermé sur $k$, il en est de même de $X$; il reste à voir que $X$ est de type fini sur $k$, mais cela résulte de [5], AC V, p. 33, Lemme 5 . De plus, d'après 5.4 , tout ensemble fini de points de $X$ est contenu dans un ouvert affine.

Cependant, $X$ n'est pas nécessairement projectif sur $k$, ainsi que le montrent les exemples suivants.

6.2. Le premier exemple est donné par le schéma obtenu en identifiant point par point deux courbes rationnelles lisses disjointes, dont les degrés dans l'espace projectif sont premiers entre eux. Plus précisement, choisissons dans l'espace projectif $\mathbb{P}_{3}$, sur un corps $k$, une droite et une conique plane, disjointes et munies d'un paramétrage; leur réunion est donc l'image d'une immersion fermée

$$
v: \mathbb{P}_{1} \sqcup \mathbb{P}_{1} \longrightarrow \mathbb{P}_{3}
$$

et on a un morphisme fini libre de rang deux évident

$$
g: \mathbb{P}_{1} \sqcup \mathbb{P}_{1} \longrightarrow \mathbb{P}_{1} .
$$

Soit $X$ le schéma obtenu en pinçant $\mathbb{P}_{3}$ par le morphisme $g$, de sorte qu'on a le carré cocartésien

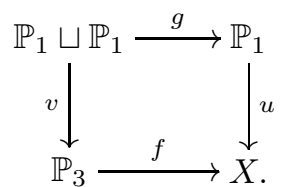

Le schéma $X$ est propre, intègre, de normalisé $\mathbb{P}_{3}$, tout ensemble fini de points de $X$ est contenu dans un ouvert affine, mais $X$ n'est pas projectif.

En effet, posons $L=\mathcal{O}_{\mathbb{P}_{1}}(1)$; par hypothèse, le faisceau $v^{\star}\left(\mathcal{O}_{\mathbb{P}_{3}}(1)\right)$ est isomorphe à $L$ sur la première composante et à $L^{\otimes 2}$ sur la seconde. Si $X$ possédait un faisceau très ample, ses images réciproques sur $\mathbb{P}_{3}$ et sur $\mathbb{P}_{1}$ seraient amples, donc respectivement de degré $a>0$ et $b>0$, et son image réciproque sur $\mathbb{P}_{1} \sqcup \mathbb{P}_{1}$ aurait le degré $a=b$ sur la première composante et le degré $2 a=b$ sur la seconde.

(Pire, on vérifie facilement qu'on a un isomorphisme $k^{\times} \stackrel{\sim}{\rightarrow} \operatorname{Pic}(X)$; l'image de $\alpha \in k^{\times}$est le faisceau inversible sur $X$ obtenu en recollant, sur $\mathbb{P}_{1} \sqcup \mathbb{P}_{1}$, le faisceau structural de $\mathbb{P}_{3}$ et celui de $\mathbb{P}_{1}$ en prenant comme isomorphisme l'identité sur la première composante et la multiplication par $\alpha \in k^{\times}$sur la seconde.)

6.3. Un second exemple est donné par le schéma obtenu en recollant deux espaces projectifs $P$ et $P^{\prime}$ le long d'un fermé «commun».

Plus précisemment, soit $A$ un schéma muni de deux immersions fermées $w: A \rightarrow P$ et $w^{\prime}: A \rightarrow P^{\prime}$; le schéma $X$ obtenu par recollement s'insère dans 
le carré cocartésien

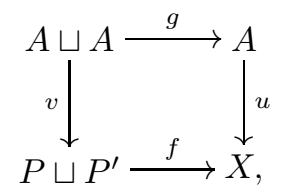

ou encore, dans le carré cocartésien d'immersions fermées

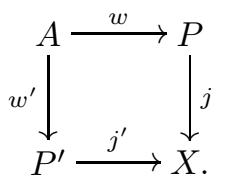

Le schéma $X$ est propre sur le corps de base et tout ensemble fini de points de $X$ est contenu dans un ouvert affine.

Désignons par $L=w^{\star}\left(\mathcal{O}_{P}(1)\right)$ et $L^{\prime}=w^{\prime \star}\left(\mathcal{O}_{P^{\prime}}(1)\right)$ les faisceaux très amples sur $A$ associés aux plongements.

Alors $X$ est projectif si et seulement si il existe deux entiers $n>0$ et $n^{\prime}>0$ et un isomorphisme $L^{\otimes n} \stackrel{\sim}{\rightarrow} L^{\prime \otimes n^{\prime}}$.

La condition est nécessaire car les images réciproques sur $P$ et sur $P^{\prime}$ d'un faisceau très ample sur $X$ sont de la forme $\mathcal{O}_{P}(n)$ et $\mathcal{O}_{P^{\prime}}\left(n^{\prime}\right)$ respectivement, avec des degrés strictement positifs.

Réciproquement, à un tel isomorphisme $L^{\otimes n} \stackrel{\sim}{\rightarrow} L^{\prime \otimes n^{\prime}}$ est associé un faisceau $M$ sur $X$, dont les restrictions à $P$ et $P^{\prime}$ sont isomorphes respectivement à $\mathcal{O}_{P}(n)$ et $\mathcal{O}_{P^{\prime}}\left(n^{\prime}\right)$; il résulte alors, par exemple de 2.2 , que $M$ est inversible, et il reste à voir qu'il est ample; or, son image réciproque par le morphisme fini surjectif $f: P \sqcup P^{\prime} \longrightarrow X$ l'est; il suffit donc d'appliquer [9], EGA III, 2.6.2.

\section{Existence de pincements ; le cas général}

L'énoncé qui suit est plus précis que 5.4 en ce qu'il donne une condition nécessaire et suffisante pour l'existence du schéma pincé; mais cette condition est difficilement vérifiable sans des hypothèses plus fortes, comme celle énoncée dans 5.4 .

THÉORÈme 7.1. - Soient $X^{\prime}$ un schéma, $Y^{\prime}$ un sous-schéma fermé de $X^{\prime}$ et $g: Y^{\prime} \rightarrow Y$ un morphisme affine. Pour tout $y \in Y$, on considère le schéma

$$
F(y)=\operatorname{Spec}\left(\mathcal{O}_{Y, y}\right) \times_{Y} Y^{\prime}=g^{-1}\left(\operatorname{Spec}\left(\mathcal{O}_{Y, y}\right)\right)
$$

A) Les conditions suivantes sont équivalentes :

BULLETIN DE LA SOCIÉTÉ MATHÉMATIQUE DE FRANCE 
i) L'espace annelé somme amalgamée $X=X^{\prime} \sqcup_{Y^{\prime}} Y$ est un schéma, et dans le carré cocartésien

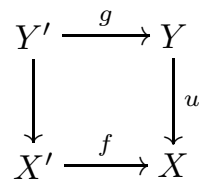

le morphisme $f$ est affine et u est une immersion fermée.

ii) Pour tout $y \in Y$, le sous-ensemble $F(y) \subset X^{\prime}$ admet une base de voisinages formée d'ouverts affines de $X^{\prime}$. De plus, si ces conditions sont vérifiées, $f$ induit un isomorphisme de $X^{\prime}-Y^{\prime}$ sur $X-Y$, le carré (7.1.1) est cartésien et le morphisme $X^{\prime} \sqcup Y \rightarrow X$ déduit de $f$ et de u est universellement submersif.

B) Supposons de plus que g soit entier; alors ces conditions sont aussi équivalentes à la suivante:

iii) Pour tout point $y \in Y$, l'ensemble $g^{-1}(y)$ est contenu dans un ouvert affine de $X^{\prime}$.

Le morphisme $f$ est alors entier.

La démonstration de 7.1 utilise les résultats suivants :

7.2.1. Soit $X$ un schéma quasi-compact et quasi-séparé. Pour qu'une partie $E$ de $X$ soit pro-constructible, il faut et il suffit qu'il existe un schéma affine $X^{\prime}$ et un morphisme $f: X^{\prime} \rightarrow X$ tels que $f\left(X^{\prime}\right)=E$; en particulier, si $E$ est fini, ou si $E$ est fermé ou si $E$ est un ouvert quasi-compact de $X$ alors $E$ est pro-constructible (voir [9], EGA I, 7.2.3 ix).

7.2.2. Soit $X$ un schéma quasi-compact, $\left(F_{i}\right)_{i \in I}$ une famille de parties proconstructibles de $X$ et $E$ une partie ind-constructible de $X$ ( par exemple un ouvert ) telles que $\bigcap_{i \in I} F_{i} \subset E$. Alors il existe une partie finie $J \subset I$ telle que $\bigcap_{j \in J} F_{J} \subset E$ (voir [9], EGA I, 7.2.5).

Montrons d'abord que la condition i) implique la dernière assertion de A) : les propriétés évoquées sont locales sur $X$, on peut donc supposer que $X$ est affine ; les trois autres schémas sont alors aussi affines. Le fait que le carré soit cartésien résulte alors de 1.3 ; comme la formation de l'espace annelé somme amalgamée commute à la restriction à un ouvert de la base, $f$ induit un isomorphisme de $f^{-1}(X-Y)$ sur $X-Y$. Montrons enfin que le morphisme $X^{\prime} \sqcup Y \rightarrow X$ est universellement submersif : il suffit de voir qu'il reste submersif après un changement de base $\bar{X} \rightarrow X$ où $\bar{X}$ est affine; désignant par le symbole surligné correspondant le schéma obtenu par ce changement de base, on a la factorisation

$$
\bar{X}^{\prime} \sqcup \bar{Y} \longrightarrow \bar{X}^{\prime} \sqcup_{\bar{Y}^{\prime}} \bar{Y} \longrightarrow \bar{X}
$$

TOME $131-2003-\mathrm{N}^{\mathrm{O}} 4$ 
où l'espace annelé du milieu est un schéma en vertu de 5.1. La flèche de gauche est submersive par définition et celle de droite est une immersion fermée définie par un idéal de carré nul d'après 2.2 , iii) ; c'est donc un homéomorphisme; d'où le résultat.

i) $\Rightarrow$ ii) : Soient $y$ un point de $Y, x=u(y)$ son image dans $X$ et posons

$$
E=\operatorname{Spec}\left(\mathcal{O}_{X, x}\right) \times_{X} X^{\prime} .
$$

Comme $f$ est affine, $E$ est un schéma affine dont l'ensemble sous-jacent s'identifie à l'intersection des ouverts affines $f^{-1}(U)$ lorsque $U$ parcourt l'ensemble des ouverts affines de $X$ contenant $x$; donc, d'après 7.2.2, $E$ admet une base de voisinages ouverts affines dans $X^{\prime}$.

D'autre part, la trace $F=E \cap Y^{\prime}$ de $E$ sur le fermé $Y^{\prime}$ s'identifie à $\operatorname{Spec}\left(\mathcal{O}_{Y, y}\right) \times_{Y} Y^{\prime}$. Il reste donc à montrer que tout ouvert de $X^{\prime}$ qui contient $F$ contient aussi $E$. Quitte à faire le changement de base plat $\operatorname{Spec}\left(\mathcal{O}_{X, x}\right) \rightarrow X$, on peut supposer que $X$ est local de point fermé $x$ et il faut alors voir que tout ouvert de $X^{\prime}$ qui contient $Y^{\prime}$ est égal à $X^{\prime}$; or, d'après 5.2, un tel ouvert contient l'image réciproque d'un ouvert du schéma local $X$ contenant le fermé non vide $Y$; cet ouvert de $X$ ne peut être que $X$ lui-même.

Pour prouver les implications ii) $\Rightarrow$ i) et iii) $\Rightarrow$ i), on se ramène à amalgamer des ouverts affines comme dans la démonstration de 5.4. Soient $y \in Y$ et $F=$ $g^{-1}\left(\operatorname{Spec}\left(\mathcal{O}_{Y, y}\right)\right)$; admettons un instant que sous chacune des hypothèses ii) et iii) on ait la propriété suivante :

(P) Il existe un ouvert affine $U^{\prime}$ de $X^{\prime}$ et un ouvert affine $V$ de $Y$ contenant $y$ tels que $F \subset U^{\prime} \cap Y^{\prime} \subset g^{-1}(V)$.

Montrons que cette propriété entraîne l'existence d'une section $s \in \Gamma\left(U^{\prime}\right)$ et d'une section $t \in \Gamma(V)$ telles que

$$
F \subset U_{s}^{\prime} \cap Y^{\prime}=g^{-1}\left(V_{t}\right),
$$

ce qui permettra d'amalgamer les ouverts affines $U_{s}^{\prime}$ et $V_{t}$.

Soit $T \subset \Gamma\left(V, \mathcal{O}_{Y}\right)$ la partie multiplicative formée des sections de $\mathcal{O}_{Y}$ définies sur $V$ et inversibles en $y$; on a

$$
F=g^{-1}\left(\operatorname{Spec}\left(\mathcal{O}_{Y, y}\right)\right)=\bigcap_{t \in T} g^{-1}\left(V_{t}\right) .
$$

D'après 7.2.2, il existe une section $t \in T$ telle que

$$
g^{-1}\left(V_{t}\right) \subset U^{\prime} \cap Y^{\prime} \subset g^{-1}(V) .
$$

Notant $t^{\prime} \in \Gamma\left(U^{\prime} \cap Y^{\prime}\right)$ l'image de la section $t$ par l'application composée

$$
\Gamma\left(V, \mathcal{O}_{Y}\right) \longrightarrow \Gamma\left(g^{-1}(V), \mathcal{O}_{Y^{\prime}}\right) \stackrel{\text { restr. }}{\longrightarrow} \Gamma\left(U^{\prime} \cap Y^{\prime}, \mathcal{O}_{Y^{\prime}}\right),
$$

on a $g^{-1}\left(V_{t}\right)=\left(U^{\prime} \cap Y^{\prime}\right)_{t^{\prime}}$. Comme $U^{\prime}$ est affine et que $U^{\prime} \cap Y^{\prime}$ est fermé dans $U^{\prime}$, l'homomorphisme $\Gamma\left(U^{\prime}, \mathcal{O}_{U^{\prime}}\right) \rightarrow \Gamma\left(U^{\prime} \cap Y^{\prime}, \mathcal{O}_{U^{\prime} \cap Y^{\prime}}\right)$ est surjectif, si bien qu'il 
existe une section $s \in \Gamma\left(U^{\prime}, \mathcal{O}_{U^{\prime}}\right)$ d'image $t^{\prime}$; bref, l'ouvert affine $U_{s}^{\prime} \subset X^{\prime}$ a la propriété requise :

$$
U_{s}^{\prime} \cap Y^{\prime}=g^{-1}\left(V_{t}\right) .
$$

Il reste donc à vérifier la propriété $(\mathrm{P})$.

Sous l'hypothèse ii), on prend n'importe quel ouvert affine $V \subset Y$ contenant $y$; l'existence d'un ouvert $U^{\prime}$ correspondant fait partie de l'hypothèse.

Supposons maintenant que $g$ soit entier et qu'il existe un ouvert affine $U^{\prime}$ de $X^{\prime}$ contenant $g^{-1}(y)$. Soit $V$ un ouvert affine de $Y$ contenant $y$; comme $g^{-1}(y)$ est un schéma affine entier sur $\operatorname{Spec}(\kappa(y))$, son anneau de sections $S=\Gamma\left(g^{-1}(y)\right)$ est réunion filtrante de ses sous- $\kappa(y)$-algèbres finies.

Soit $J \subset \Gamma\left(U^{\prime} \cap Y^{\prime}\right)$ un idéal définissant l'ouvert $U^{\prime} \cap g^{-1}(V) \subset U^{\prime} \cap Y^{\prime}$. En désignant par

$$
\rho: \Gamma\left(U^{\prime} \cap Y^{\prime}\right) \longrightarrow S=\Gamma\left(g^{-1}(y)\right)
$$

l'homomorphisme de restriction, on a $\rho(J) S=S$ puisque $g^{-1}(y)$ est contenu dans l'ouvert $U^{\prime} \cap g^{-1}(V)$ que définit $J$; cela s'écrit aussi $\Sigma \rho\left(t_{i}\right) s_{i}=1$, avec $t_{i} \in J$ et $s_{i} \in S$. Soit $R \subset S$ une sous- $\kappa(y)$-algèbres finie contenant les $s_{i}$ et les $\rho\left(t_{i}\right)$, et soit $A=\rho^{-1}(R)$, de sorte qu'on a le diagramme suivant :

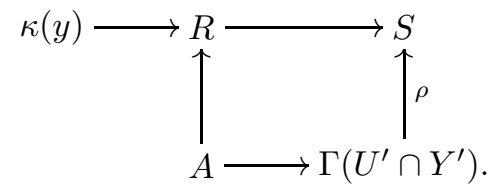

L'idéal a de $A$ engendré par les $t_{i}$ vérifie, par construction, la relation $\rho(\mathfrak{a}) R=R$; mais l'anneau $R$ est un produit fini d'anneaux locaux artiniens, puisqu'il est fini sur le corps $\kappa(y)$; il existe donc un élément $t \in \mathfrak{a}$ tel que $\rho(t)$ soit inversible dans $R$; comme $t \in \mathfrak{a} \subset J$, on a les inclusions

$$
g^{-1}(y) \subset\left(U^{\prime} \cap Y^{\prime}\right)_{t} \subset U^{\prime} \cap g^{-1}(V) .
$$

Finalement, en relevant $t$ en une section $s \in \Gamma\left(U^{\prime}\right)$ on obtient des ouverts $U_{s}^{\prime}$ et $V$ qui vérifient la propriété $(\mathrm{P})$.

L'assertion d'existence du théorème 7.1 est ainsi démontrée. Le fait que $f$ soit entier lorsque $g$ l'est a déjà été signalé dans 5.6,3).

7.3. L'exemple qui suit, suggéré par P. Samuel, repose sur une situation où un fermé $Y^{\prime}$ est affine et n'est contenu dans aucun ouvert affine de $X^{\prime}$.

Soient $\mathbb{A}=\mathbb{C}^{2}$ le plan complexe affine et $E \subset \mathbb{A}$ une courbe elliptique (du moins sa partie affine). Étant isomorphe au quotient de $\mathbb{C}$ par un réseau, le groupe $\operatorname{Pic}(E)$ n'est pas de torsion; comme il est engendré par les classes des faisceaux d'idéaux de $\mathcal{O}_{E}$ correspondants aux points fermés, il existe un point $p \in E$ tel que l'idéal correspondant $\mathfrak{m} \subset \Gamma\left(E, \mathcal{O}_{E}\right)$ n'ait aucune puissance principale. Il n'existe donc pas de section $t \in \Gamma\left(E, \mathcal{O}_{E}\right)$ telle que $E_{t}=E-\{p\}$ (On utilise le fait que dans l'anneau de Dedekind $\Gamma\left(E, \mathcal{O}_{E}\right)$ l'idéal non nul $(t)$ 
est produit d'idéaux maximaux.) Posons $X^{\prime}=\mathbb{A}-\{p\}$, et $Y^{\prime}=E-\{p\}$. Comme l'anneau $\mathbb{C}\left[X_{1}, X_{2}\right]$ est factoriel, tout ouvert affine de $X^{\prime}$ est défini par une section; par suite, aucun ouvert affine de $X^{\prime}$ ne contient le fermé affine $Y^{\prime}$. Prenant pour morphisme $g: Y^{\prime} \rightarrow Y$ le morphisme (affine) $Y^{\prime} \rightarrow \operatorname{Spec}(\mathbb{C})$, la condition ii) de 7.1 ne sera donc pas vérifiée.

Cependant, l'espace annelé « pincé » $X$ est un schéma affine! (Cela entraîne que le morphisme $f: X^{\prime} \rightarrow X$ n'est pas affine, puisque $X^{\prime}$ n'est pas un schéma affine.)

Pour voir tout cela, considérons le schéma affine $\bar{X}$ obtenu en pinçant dans $\mathbb{A}$ toute la courbe $E$ en un point (5.1). On peut montrer que le morphisme d'espaces annelés

$$
\varphi: X \longrightarrow \bar{X}
$$

déduit de la propriété universelle de la somme amalgamée, est un isomorphisme. Signalons seulement que le point clé est le suivant :

Pour tout ouvert $U$ du plan $\mathbb{A}$, contenant $E-p$, l'ensemble $U \cup p$ est ouvert et l'homomorphisme de restriction

$$
\Gamma\left(U \cup p, \mathcal{O}_{\mathbb{A}}\right) \longrightarrow \Gamma\left(U, \mathcal{O}_{\mathbb{A}}\right)
$$

est un isomorphisme.

La seconde propriété provient de ce que $\operatorname{prof}\left(\mathcal{O}_{\mathbb{A}, p}\right) \geq 2$. Pour vérifier que $U \cup p$ est ouvert, on peut supposer que $p \notin U$ et donc, notant $F$ le fermé complémentaire de $U$, que l'on a $F \cap E=p$. Ce fermé se décompose en $F=$ $F_{0} \cup F_{1}$, où $F_{0}$ est une réunion finie de points fermés et où $F_{1}$ est purement de codimension 1 dans $\mathbb{A}$. Comme $\mathbb{A}$ est factoriel, le fermé $F_{1}$ est défini par une équation; mais, par hypothèse, $p$ ne peut pas être défini dans $E$ par l'annulation d'une seule équation; on voit donc que $F_{1} \cap E=\varnothing$, donc que $p$ est un des points de $F_{0}$; cela montre que l'ensemble $U \cup p$ est ouvert puisqu'il est le complémentaire de $\left(F_{0}-p\right) \cup F_{1}$.

7.4. Complément. — Nous laissons au lecteur le soin d'énoncer et de vérifier un analogue de 2.2 portant sur le produit fibré des catégories de faisceaux quasicohérents sur les schémas en jeu.

\section{Applications aux monomorphismes}

Voici des applications des résultats qui précèdent, tout à fait dans l'esprit du séminaire organisé par P. Samuel [18] en 68/69.

BULlETIN DE LA SOCIÉtÉ MATHÉMATIQUE DE FRANCE 
8.1. Rappelons qu'un morphisme de schémas $f: X^{\prime} \rightarrow X$ est un monomorphisme si pour tout couple de morphismes de schémas $X^{\prime \prime} \underset{v}{\stackrel{u}{\rightleftarrows}} X^{\prime}$, la relation $f u=f v$ implique $u=v$; il revient au même de dire que le morphisme diagonal $\Delta_{f}: X^{\prime} \rightarrow X^{\prime} \times_{X} X^{\prime}$ est un isomorphisme. Les immersions sont des monomorphismes, ainsi que les morphismes $\operatorname{Spec}\left(S^{-1} A\right) \rightarrow \operatorname{Spec}(A)$ associés aux anneaux de fractions; si $F$ est un sous-schéma fermé de $X$ et si $U$ désigne l'ouvert complémentaire, alors le morphisme $F \sqcup U \rightarrow X$ est un monomorphisme surjectif.

Pour qu'un morphisme de schémas $f: X^{\prime} \rightarrow X$ soit un monomorphisme plat, il faut et il suffit que $f$ soit injectif et que, pour tout $x^{\prime} \in X^{\prime}$, l'homomorphisme $\mathcal{O}_{X, f\left(x^{\prime}\right)} \rightarrow \mathcal{O}_{X^{\prime}, x^{\prime}}$ soit un isomorphisme.

Les points doubles ordinaires des courbes conduisent à des monomorphismes : en effet, soit $C$ une courbe intègre, de normalisée $C^{\prime}$; supposons que le seul point singulier de $C$ soit un point double, dont l'image réciproque dans $C^{\prime}$ soit donc constituée de deux points distincts $y$ et $z$; alors le morphisme $C^{\prime}-\{z\} \rightarrow C$ est un monomorphisme. Plus généralement,

Proposition 8.2. - Soient $Y$ et $Z$ deux sous-schémas fermés disjoints d'un schéma $X^{\prime}$. On suppose donné un isomorphisme $\gamma: Z \rightarrow Y$ et on note $g$ : $Y \sqcup Z \rightarrow Y$ le morphisme défini par $\operatorname{id}_{Y}$ et $\gamma$. On suppose enfin que pour tout point $z \in Z$, un ouvert affine de $X^{\prime}$ contient à la fois $z$ et $\gamma(z)$, de sorte qu'on peut «pincer» $X^{\prime}$ en identifiant chaque point $z$ de $Z$ à $\gamma(z)$. Le schéma $X$ obtenu s'insère dans le carré cocartésien

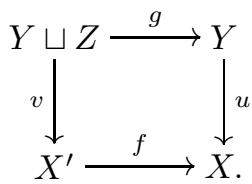

Alors, la restriction de $f$ à l'ouvert $V=X^{\prime}-Z$ définit un monomorphisme $h: V \rightarrow X$ qui est surjectif et de type fini.

Posons $Y^{\prime}=Y \sqcup Z$. D'après 5.6, 1, le carré d'immersions fermées

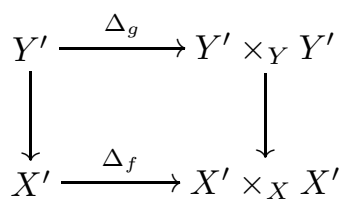

est cocartésien; sa restriction à l'ouvert $V \times_{X} V \subset X^{\prime} \times_{X} X^{\prime}$ l'est encore; mais la restriction de $V \times_{X} V$ à la diagonale $X^{\prime}$ est égale à la diagonale $V$; de plus, comme $V=X^{\prime}-Z$, on a $\left(V \times_{X} V\right) \cap\left(Y^{\prime} \times_{Y} Y^{\prime}\right)=Y \times_{Y} Y$; par suite, puisque 
le carré

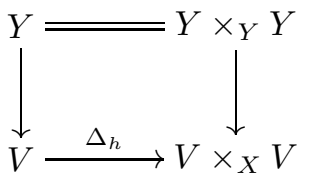

est cocartésien, $\Delta_{h}$ est un isomorphisme.

8.3. Rappelons qu'un schéma est dit absolument plat si tous ses anneaux locaux sont des corps et qu'à tout schéma $X$ est associé un schéma absolument plat universel, noté ici $T(X)$; Grothendieck, dans [9], EGA I, 7.2.14, l'annonce à mots couverts et le note $X^{\text {cons }}$, car son espace topologique sous-jacent est homéomorphe à $X$ muni de la topologie constructible. J.-P. Olivier le construit explicitement dans l'exposé 6 du séminaire Samuel et dans [13]; il montre en particulier que le morphisme canonique $t_{X}: T(X) \rightarrow X$ est un monomorphisme affine et surjectif.

On va utiliser cette construction et un pincement pour démontrer que tout monomorphisme quasi-compact $u: Y \rightarrow X$ se factorise en $Y \stackrel{j}{\rightarrow} Z \stackrel{v}{\rightarrow} X$, où $j$ est une immersion fermée plate et $v$ un monomorphisme quasi-compact surjectif.

Les énoncés 8.4 et 8.5 qui suivent sont essentiellement extraits de [5], AC II, p. 173 , exercices 16 et 17 .

Lemme 8.4. - Soit $\left(X, \mathcal{O}_{X}\right)$ un espace annelé tel que pour tout $x \in X, \mathcal{O}_{X, x}$ soit un corps. Alors l'anneau $\Gamma\left(X, \mathcal{O}_{X}\right)$ est absolument plat.

Il s'agit de montrer que tout élément $f \in A=\Gamma\left(X, \mathcal{O}_{X}\right)$ engendre un idéal idempotent de $A$. Or, comme les anneaux locaux de $X$ sont des corps, l'ouvert $X_{f}$ formé des points $x$ tels que l'image de $f$ soit inversible dans $\mathcal{O}_{X, x}$, est égal à l'ensemble des points où l'image de $f$ est non nulle, c'est-à-dire au support de $f$; l'ensemble $X_{f}$ est donc une partie à la fois ouverte et fermée de $X$; par suite, l'homomorphisme canonique $A \rightarrow \Gamma\left(X_{f}, \mathcal{O}_{X}\right) \times \Gamma\left(X-X_{f}, \mathcal{O}_{X}\right)$ est un isomorphisme; comme l'image de $f$ dans le premier facteur est inversible par définition de $X_{f}$, il existe une section $g \in A$ dont la restriction à $X_{f}$ est l'inverse de celle de $f$; la section $f-g f^{2}$ a donc une restriction nulle au dessus de $X_{f}$; comme $f$ a une restriction nulle sur $X-X_{f}$, on voit que $f=g f^{2}$, donc que $f A=f^{2} A$.

Proposition 8.5. - Soit $\left(X, \mathcal{O}_{X}\right)$ un espace annelé tel que pour tout $x \in X$, $\mathcal{O}_{X, x}$ soit un corps. Les propriétés suivantes sont équivalentes :

i) $X$ est un schéma affine.

ii) L'espace topologique $X$ est compact et totalement discontinu.

iii) $X$ est un schéma quasi-compact et quasi-séparé.

i) $\Rightarrow$ ii). Supposons que $X$ soit un schéma affine d'anneau $A$ et montrons d'abord que $X$ est séparé (au sens topologique). Soient $\mathfrak{m}$ et $\mathfrak{n}$ deux idéaux BULLETIN DE LA SOCIÉTÉ MATHÉMATIQUE DE FRANCE 
premiers distincts ; comme, par hypothèse, les localisés $A_{\mathfrak{m}}$ et $A_{\mathfrak{n}}$ sont des corps, ces idéaux premiers sont minimaux; par suite, on a $A_{\mathfrak{m}} \otimes_{A} A_{\mathfrak{n}}=0$; il existe donc des éléments $f \in A-\mathfrak{m}$ et $g \in A-\mathfrak{n}$ tels que $A_{f} \otimes_{A} A_{g}=0$; cela signifie que les ouverts $\mathrm{D}(f)$ et $\mathrm{D}(g)$ séparent les points considérés; $X$ est donc compact. Par ailleurs, le lemme 8.4 montre que dans l'anneau $A$ on a $f A=f^{2} A$ pour tout $f$; autrement dit, les ouverts $\mathrm{D}(f)$ sont aussi fermés; comme l'intersection de ceux qui contiennent un point est réduite à ce point, on voit que la composante connexe d'un point de $X$ est réduite à ce point, ce qu'on exprime en disant que $X$ est totalement discontinu.

ii) $\Rightarrow$ i). Considérons le morphisme d'espaces localement annelés associé à l'application identique $\Gamma\left(S, \mathcal{O}_{S}\right)=A=\Gamma\left(X, \mathcal{O}_{X}\right)(\operatorname{voir}[9]$, EGA I, 1.6.3)

$$
u: X \longrightarrow S=\operatorname{Spec}(A) .
$$

Il s'agit de voir que c'est un isomorphisme lorsque $X$ est compact et totalement discontinu. La compacité implique que la composante connexe d'un point $x$ est égale à l'intersection des parties ouvertes et fermées le contenant [6] TG II, $\S 4$, prop. 6 , p. 32 , et la seconde hypothèse entraine que cette intersection est réduite à $x$.

Par ailleurs, à une partie ouverte et fermée $U \subset X$ est associée une décomposition en produit

$$
A=\Gamma\left(X, \mathcal{O}_{X}\right) \simeq \Gamma\left(U, \mathcal{O}_{X}\right) \times \Gamma\left(X-U, \mathcal{O}_{X}\right),
$$

et donc un unique idempotent $f \in A$ tel que l'on ait $U=X_{f}$ et un isomorphisme

$$
A_{f} \simeq \Gamma\left(U, \mathcal{O}_{X}\right) .
$$

Montrons que $u$ est injectif : soient $x$ et $y$ des points distincts de $X$; par hypothèse, il existe un ouvert et fermé de $X$ contenant $x$ et ne contenant pas $y$; d'après ce qui précède, il existe donc une section $f \in A$ telle que $x \in X_{f}$ et $y \notin X_{f} ;$ comme $u^{-1}\left(S_{f}\right)=X_{f}$, on a $u(x) \in S_{f}$ et $u(y) \notin S_{f}$.

Montrons que $u$ est surjective. Il résulte de (8.4) et de l'implication i) $\Rightarrow$ ii) que $S$ est lui-aussi compact et totalement discontinu; en particulier, tout point $s \in S$ est l'intersection d'une famille $\left(S_{f}\right)_{f \in F}$ d'ouverts spéciaux; il faut montrer que l'intersection des $X_{f}$ est non vide; or, chacun d'eux est visiblement non vide, la famille $\left(X_{f}\right)_{f \in F}$ est filtrante et enfin $X$ est compact.

Ainsi, l'application $u$ est-elle une bijection continue entre espaces compacts ; c'est donc un homéomorphisme. Par ailleurs, pour $f$ dans l'anneau absolument plat $A$, l'ouvert $S_{f}$ est aussi fermé, donc $X_{f}=u\left(S_{f}\right)$ est ouvert et fermé dans $X$; par suite, l'application

$$
\Gamma\left(S_{f}, \mathcal{O}_{S}\right)=A_{f} \longrightarrow \Gamma\left(X_{f}, \mathcal{O}_{X}\right)
$$

est un isomorphisme; comme ces ouverts $S_{f}$ forment une base pour la topologie de $S$, on voit que $u$ induit un isomorphisme de faisceaux; $u$ est donc un isomorphisme d'espaces annelés. 
L'implication i) $\Rightarrow$ iii) vient de ce qu'un schéma affine est quasi-compact et quasi-séparé.

Il reste donc à démontrer l'implication réciproque iii) $\Rightarrow$ i). Montrons d'abord que tout ouvert quasi-compact du schéma $X$ est fermé. Soient donc $x \in \bar{U}$ un point adhérent à $U$ et $V$ un ouvert affine de $X$ contenant $x$. Comme $X$ est quasi-séparé, $U \cap V$ est quasi-compact; or, l'implication i) $\Rightarrow$ ii) montre que $V$ est compact, donc que $U \cap V$ est fermé dans $V[6]$ TG I, $\S 9$, prop. 4, p. 62; par suite, $x$ est dans $U$.

Comme le schéma $X$ est quasi-compact, il est réunion finie d'ouverts affines; il suffit donc, pour pouvoir conclure, de montrer que la réunion de deux ouverts affines $U$ et $V$ est encore affine; or, on vient de voir que $U \cap V$ est ouvert et fermé, puisqu'il est quasi-compact; son complémentaire $W=V-U \cap V$ est donc ouvert et fermé dans le schéma affine $V$; c'est donc lui-même un ouvert affine; par suite, $U \cup V=U \sqcup W$ est affine.

Lemme 8.6. - Soit u $: X \rightarrow S$ un monomorphisme quasi-compact de schémas. Si $S$ est absolument plat, u est une immersion fermée plate.

On peut supposer que $S$ est affine ; par suite, $X$ est quasi-compact et séparé. Comme $S$ est absolument plat, $u$ est plat; cela entraîne que pour tout $x \in X$, l'homomorphisme $\mathcal{O}_{S, u(x)} \rightarrow \mathcal{O}_{X, x}$ est un isomorphisme; l'anneau local $\mathcal{O}_{X, x}$ est donc un corps; par suite, d'après $8.5, X$ est affine. Posons $A=\Gamma\left(S, \mathcal{O}_{S}\right)$ et $B=\Gamma\left(X, \mathcal{O}_{X}\right)$; soit $I$ le noyau de l'homomorphisme $A \rightarrow B$ associé à $u$; le schéma $S^{\prime}=\operatorname{Spec}(A / I)$ est absolument plat, donc $X \rightarrow S^{\prime}$ est surjectif; comme c'est aussi un monomorphisme plat quasi-compact, c'est un isomorphisme; donc $u$ est une immersion fermée plate.

Proposition 8.7. - Un monomorphisme $u: Y \rightarrow X$ quasi-compact de schémas se factorise en

$$
Y \stackrel{j}{\longrightarrow} Z \stackrel{v}{\longrightarrow} X
$$

où $j$ est une immersion fermée plate et $v$ un monomorphisme quasi-compact surjectif.

Considérons le diagramme commutatif

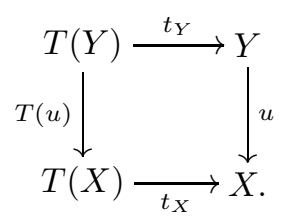

Comme $T(X)$ est absolument plat, $T(u)$ est une immersion fermée plate d'après 8.6. Par définition, les morphismes $t_{X}$ et $t_{Y}$ sont affines. On va montrer que l'espace annelé $Z=T(X) \sqcup_{T(Y)} Y$ est un schéma en utilisant le critère 7.1. 
Soient $y$ un point de $Y$ et $U^{\prime}$ un ouvert de $T(X)$ contenant $F=$ $\operatorname{Spec}\left(\mathcal{O}_{Y, y}\right) \times_{Y} T(Y)=t_{Y}{ }^{-1}\left(\operatorname{Spec}\left(\mathcal{O}_{Y, y}\right)\right)$; il s'agit de montrer qu'il existe un ouvert affine $U$ de $T(X)$ tel que $F \subset U \subset U^{\prime}$; or, $t_{Y}$ étant un morphisme affine, le schéma $F$ est affine donc quasi-compact; par suite, il existe un ouvert quasi-compact $U$ de $T(X)$ tel que $F \subset U \subset U^{\prime}$; mais $U$ est alors affine d'après 8.5. On voit donc que $Z$ est un schéma et que dans le diagramme commutatif

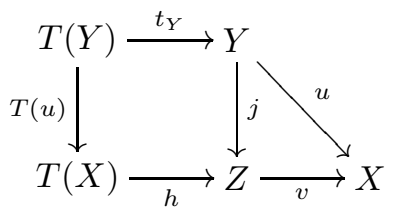

$j$ est une immersion fermée, $h$ est affine et induit un isomorphisme de $V^{\prime}=$ $T(X)-T(Y)$ sur $V=Z-Y$; enfin, comme $t_{Y}$ induit une bijection d'ensembles, il en est de même de $h$; on en déduit déjà que $v$ est radiciel puisque $t_{X}=v h$ est un monomorphisme. Montrons que $j$ est plat : en vertu de 2.2, iv), il suffit de vérifier qu'il le devient après les changements de base par $j$ et par $h$; or $Y \times{ }_{Z} Y \rightarrow Y$ est un isomorphisme puisque $j$ est une immersion fermée, et d'autre part le carré est cartésien et $T(X)$ est un schéma absolument plat.

Il reste à montrer que $v$ est un monomorphisme quasi-compact et surjectif. Le fait que $v$ soit bijectif et quasi-compact découle de ce que $t_{X}=v h$ est un monomorphisme surjectif et affine - donc quasi-compact -, et de ce que $h$ est surjectif. Il reste à vérifier que, pour tout $z \in Z$, l'homomorphisme $\mathcal{O}_{X, v z} \rightarrow \mathcal{O}_{Z, z}$ est un épimorphisme. Or, si $z \notin j(Y)$, il existe un unique point $x^{\prime} \in T(X)$ d'image $z$ et l'homomorphisme $\mathcal{O}_{Z, z} \rightarrow \mathcal{O}_{T(X), x^{\prime}}$ est un isomorphisme et on peut conclure puisque l'homomorphisme composé

$$
\mathcal{O}_{X, v z} \longrightarrow \mathcal{O}_{Z, z} \stackrel{\sim}{\longrightarrow} \mathcal{O}_{X^{\prime}, x^{\prime}}
$$

est un épimorphisme. Maintenant, si $z=j(y)$, avec $y \in Y$, alors l'homomorphisme $\mathcal{O}_{Z, z} \rightarrow \mathcal{O}_{Y, y}$ est une surjection plate d'anneaux locaux, donc est un isomorphisme et le composé

$$
\mathcal{O}_{X, v z} \longrightarrow \mathcal{O}_{Z, z} \stackrel{\sim}{\longrightarrow} \mathcal{O}_{Y, y}
$$

est un épimorphisme par l'hypothèse faite sur $u$.

\section{BIBLIOGRAPHIE}

[1] Anantharaman (S.) - Schémas en groupes, espaces homogènes et espaces algébriques sur une base de dimension 1, Mém. Soc. Math. France, vol. 33, Soc. Math. France, Paris, 1973.

[2] Artin (M.) - Algebraization of Formal Moduli II : Existence of Modifications, Annals of Math., t. 91 (1970), pp. 88-135. 
[3] Bass (H.) - Algebraic K-Theory, Benjamin, 1968.

[4] Bénabou (J.) \& Roubaud (J.) - Monades et descente, C. R. Acad. Sci. Paris, t. 270 (1970), pp. 96-98.

[5] Bourbaki (N.) - Algèbre commutative, Masson, Paris, 1961-1998.

[6] — Topologie générale, chap. 1 à 4, Masson, Paris, 1990.

[7] Colmez (P.) \& Serre (J.-P.), éds. - Correspondance GrothendieckSerre, Documents mathématiques, vol. 2, Soc. Math. France, Paris, 2001.

[8] Ferrand (D.) - Descente de la platitude par un homomorphisme fini, C. R. Acad. Sci. Paris, t. 269 (1969), pp. 946-949.

[9] Grothendieck (A.) - Éléments de géométrie algébrique, rédigés avec la collaboration de J. Dieudonné, chap. I, Springer-Verlag, 1971; chap. II-IV, Publ. Math. I.H.E.S., 1961-1967.

[10] - Techniques de descente et théorèmes d'existence en géométrie algébrique, I, in Séminaire Bourbaki, 1959/60, Soc. Math. France, Paris, 1995, exposé 190.

[11] Levelt (A.H.M.) - Sur la pro-représentabilité de certains foncteurs en géométrie algébrique, Katholieke Universiteit, Nijmegen, 1964, notes multigraphiées.

[12] Milnor (J.) - Introduction to Algebraic K-Theory, Ann. Math. Studies, vol. 72, Princeton University Press, 1971.

[13] Olivier (J.-P.) - Anneaux absolument plats universels, in [18], exposé 6.

[14] L'anneau absolument plat universel, les épimorphismes et les parties constructibles, Bol. Soc. Mat. Mexicana, t. 23 (1978), no. 2, pp. 68-74.

[15] Philippe (A.) - Morphisme net d'anneaux et descente, Bull. Sci. Math., t. 97 (1973), no. 2, pp. 57-64.

[16] Raynaud (M.) - Un critère d'effectivité de descente, in [18], exposé 5.

[17] _ Passage au quotient par une relation d'équivalence plate, in Proc. Conf. on Local Fields, Springer-Verlag, 1967, pp. 78-85.

[18] Samuel (P.), éd. - Séminaire d'algèbre commutative, 1967/68 : Les épimorphismes d'anneaux, E.N.S.J.F., 1968.

[19] Serre (J.-P.) - Groupes algébriques et corps de classes, Hermann, Paris, 1959.

[20] Venken (J.) - Non effectivité de la descente de modules plats par un morphisme fini d'anneaux locaux artiniens, C. R. Acad. Sci. Paris, Sér. A-B, t. 272 (1971), pp. A1553-A1554. 